\title{
Climate change will cause non-analogue vegetation states in Africa and commit vegetation to long-term change
}

Mirjam Pfeiffer ${ }^{1}$, Dushyant Kumar ${ }^{1}$, Carola Martens ${ }^{2}$, and Simon Scheiter ${ }^{1}$

${ }^{1}$ Senckenberg Biodiversity and Climate Research Centre (BiK-F), Senckenberganlage 25, 60438 Frankfurt am Main, Germany

${ }^{2}$ Insitute of Physical Geography, Goethe University Frankfurt am Main, Altenhoeferallee 1, 60438 Frankfurt am Main, Germany

Correspondence: Mirjam Pfeiffer (mirjam.pfeiffer@senckenberg.de)

\section{SUPPLEMENT A}

Supplementary table and figures referenced in the publication.

Table S1. Criteria used to define biome boundaries based on grid-cell level values of key variables

\begin{tabular}{lcccc}
\hline Biome type & $\begin{array}{c}\text { Grass } \\
\text { biomass [t/ha] } \\
{[\mathrm{t} / \mathrm{ha}]}\end{array}$ & $\begin{array}{c}\mathrm{C}_{3}: \mathrm{C}_{4} \\
\text { grass } \\
\text { ratio }\end{array}$ & $\begin{array}{c}\text { Total tree [\%] } \\
\text { cover } \\
{[\%]}\end{array}$ & $\begin{array}{c}\text { Forest:Savanna } \\
\text { tree cover } \\
\text { ratio }\end{array}$ \\
\hline Desert & $\leq 0.5$ & - & $\leq 10$ & - \\
$\mathrm{C}_{3}$ grassland & $>0.5$ & $>0.5$ & $\leq 10$ & - \\
$\mathrm{C}_{4}$ grassland & $>0.5$ & $<=0.5$ & $\leq 10$ & - \\
$\mathrm{C}_{3}$ savanna & - & $>0.5$ & $10-80$ & $\leq 0.5$ \\
$\mathrm{C}_{3}$ savanna & - & $\leq 0.5$ & $10-80$ & $\leq 0.5$ \\
Woodland & - & - & $10-80$ & $>0.5$ \\
Forest & - & - & $>80$ & - \\
\hline
\end{tabular}



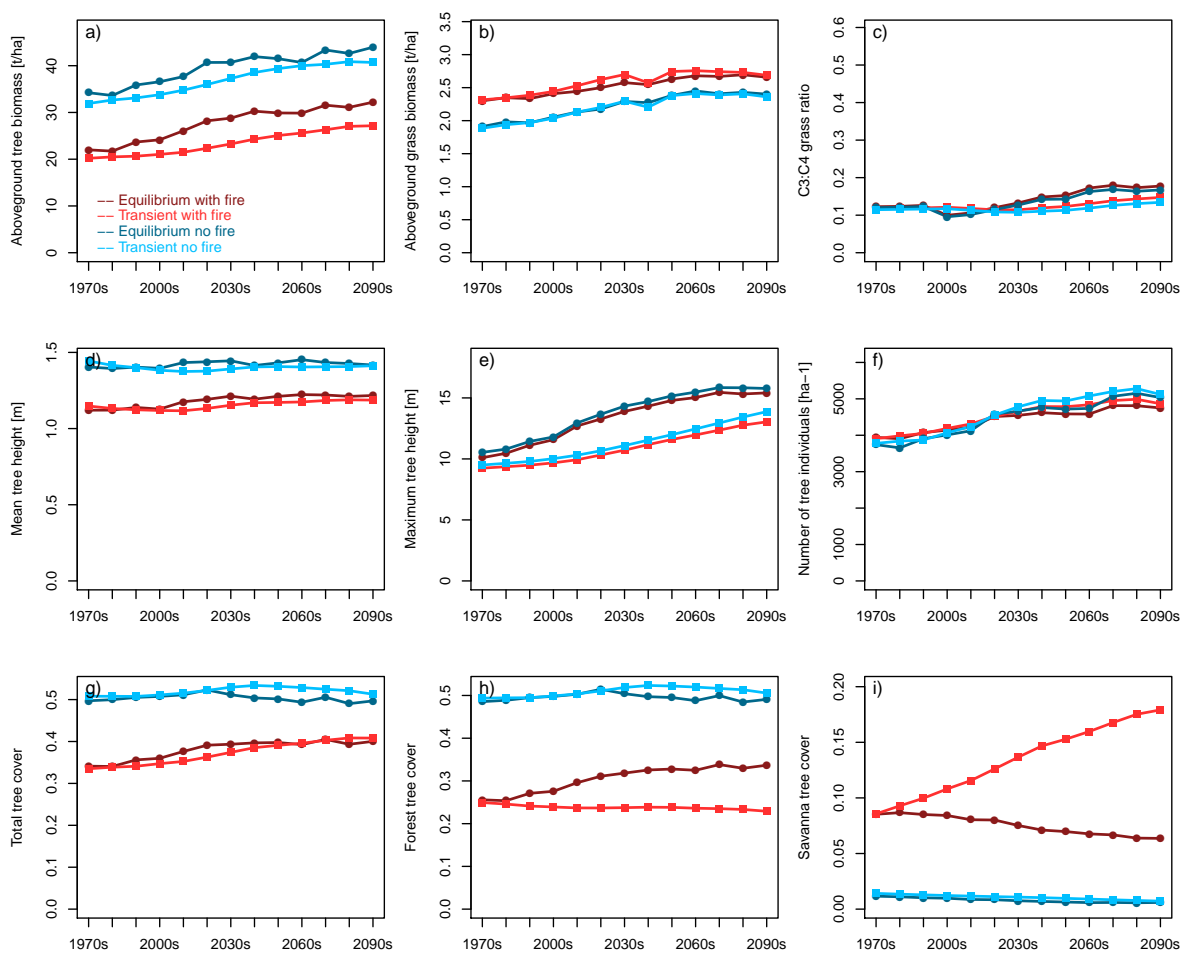

Figure S1. Time series of continental-scale spatial averages of variables for RCP4.5, calculated from decadal averages of grid cells. 

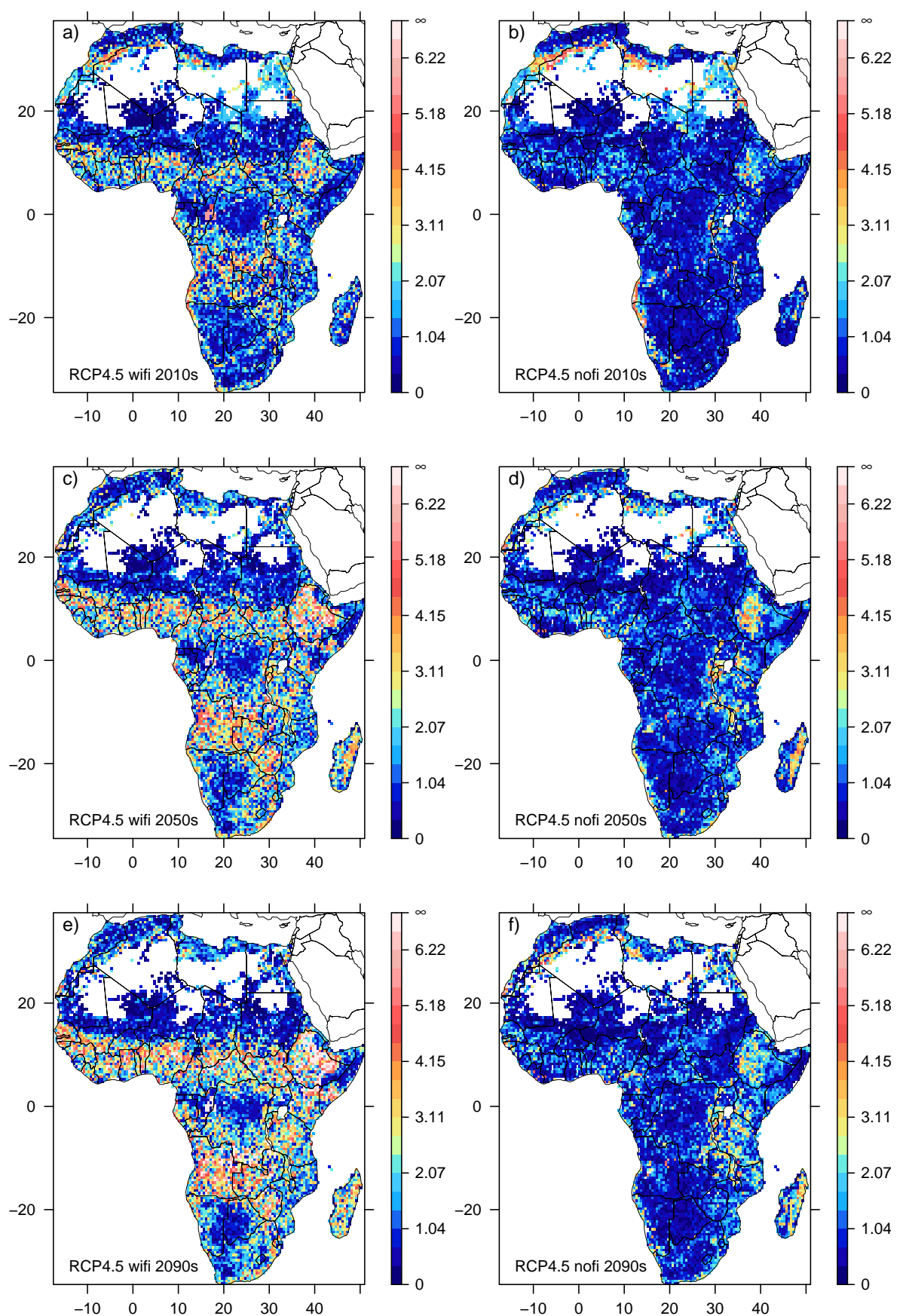

Figure S2. Spatial patterns of Euclidean distance between SDPs in RCP4.5 for three selected decades (2010-2019, 2050-2059, 2090-2099). Panels a, $\mathrm{c}$, and e represent distance between SDPs in simulations including fire, panels $\mathrm{b}, \mathrm{d}$ and $\mathrm{f}$ are for SDPs from simulations excluding fire. 

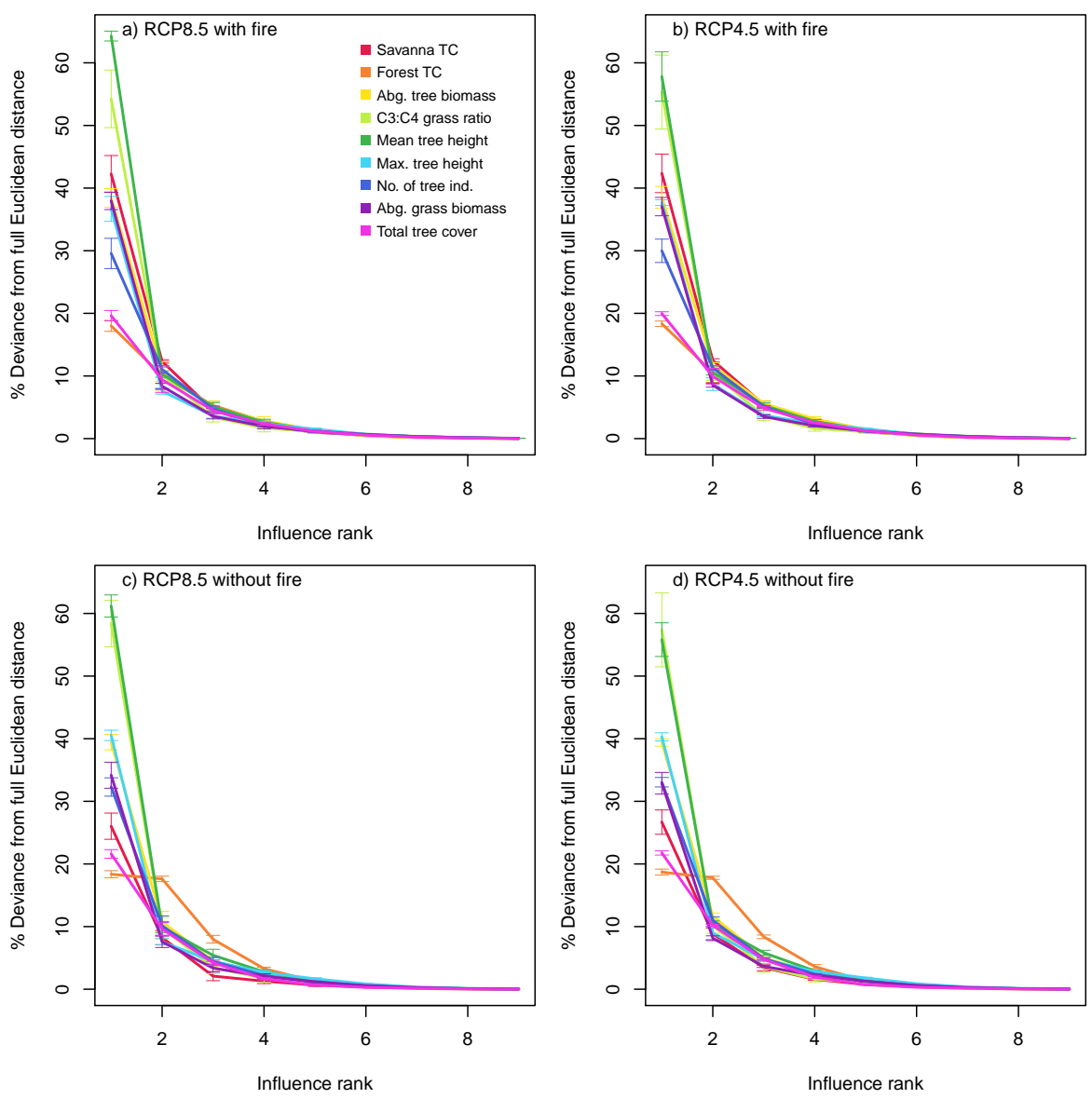

Figure S3. Decay of influence on full Euclidean distance between SDPs according to variable rank, and separated by variable identity. The second-most influential variable had substantially less influence on full Euclidean distance than the dominant variable, and influence of higher-ranked variables rapidly declined. 

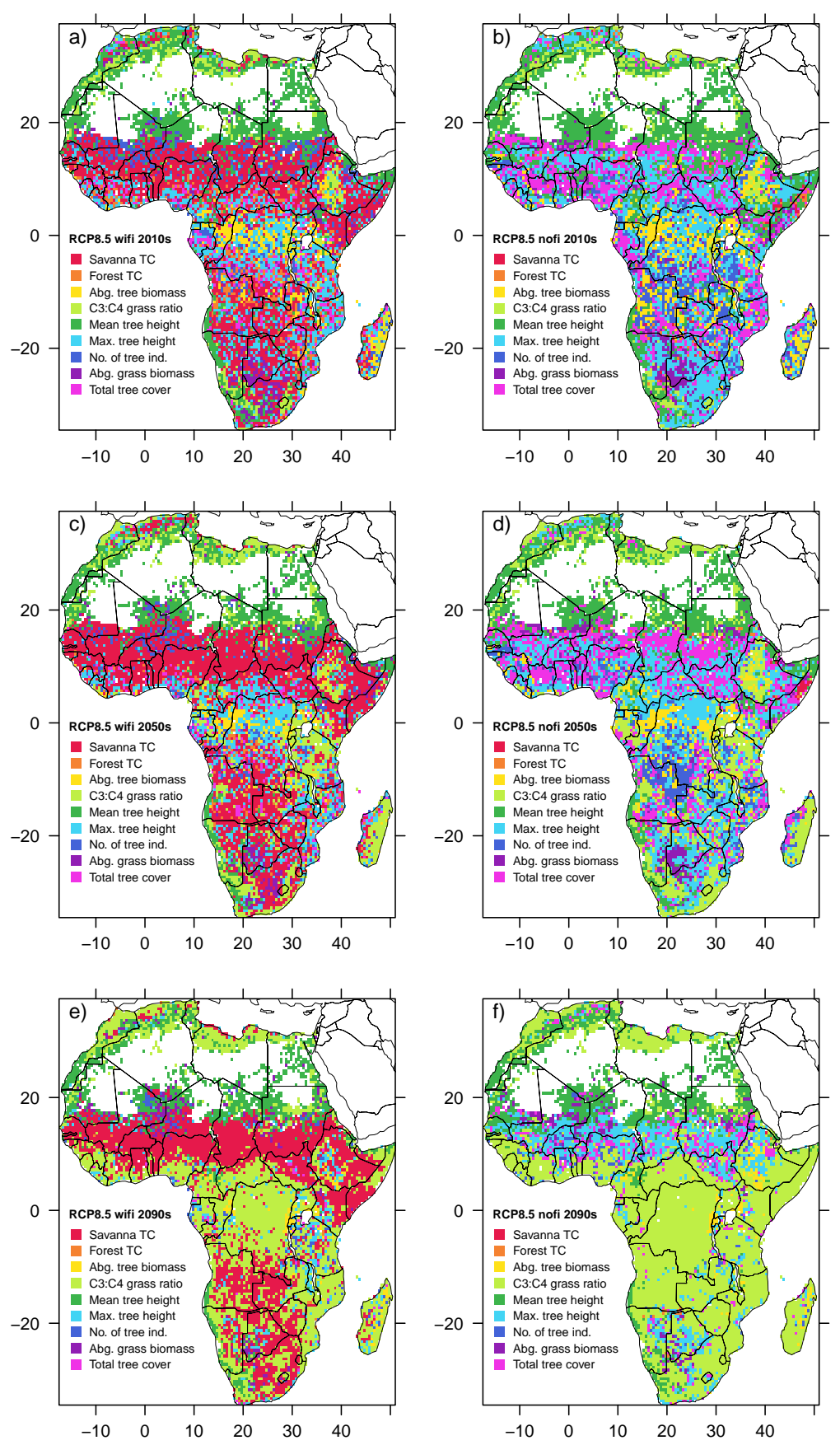

Figure S4. Spatial pattern of dominant variables with respect to Euclidean distance between SDPs for RCP8.5 for the three reference decades. Panels a, c, and e show the spatial pattern for RCP8.5 with fire, panels b, d, and f the pattern for RCP8.5 without fire. 

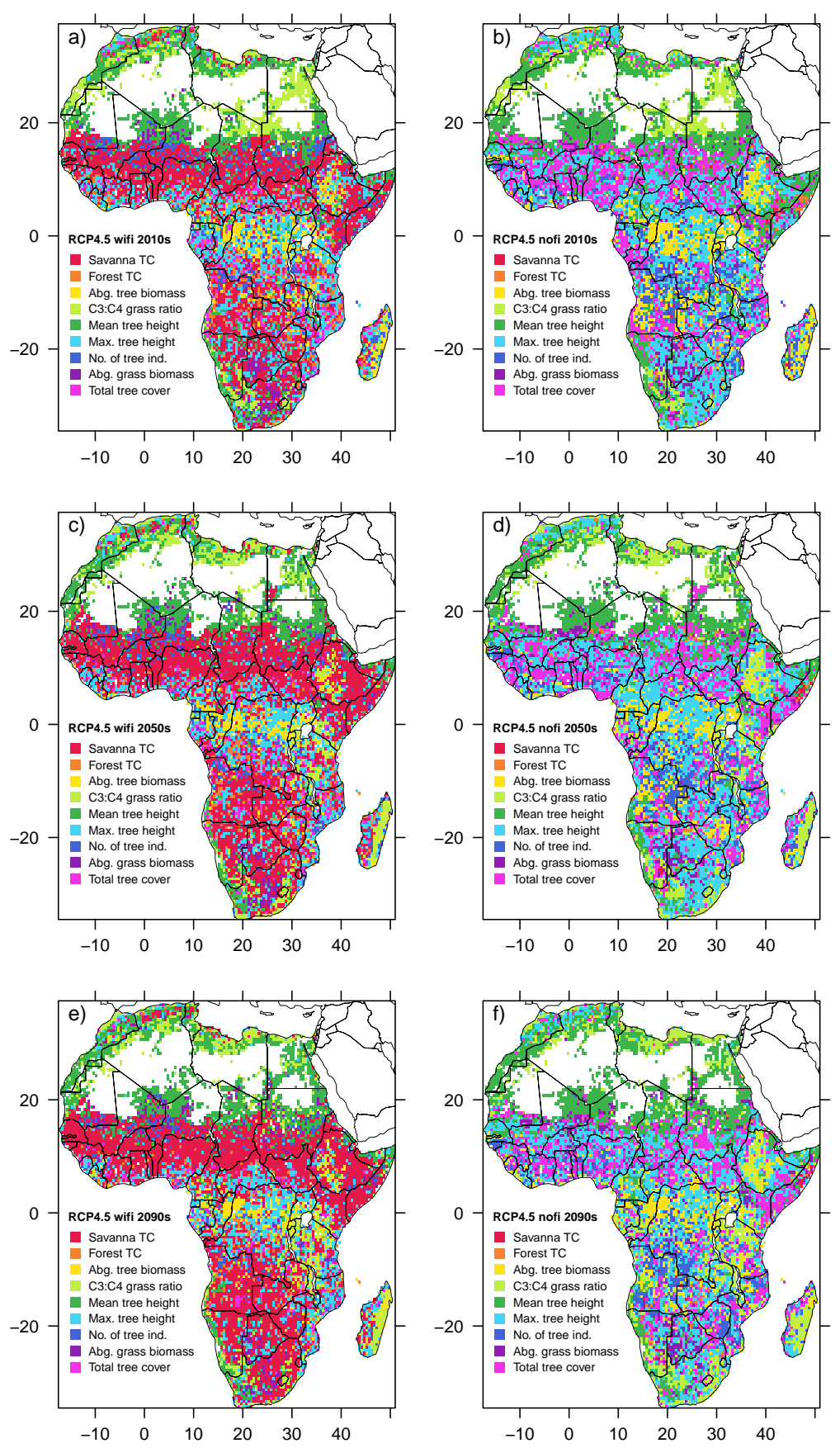

Figure S5. Spatial pattern of dominant variables with respect to Euclidean distance between SDPs for RCP4.5 for the three reference decades. Panels a, c, and e show the spatial pattern for RCP4.5 with fire, panels b, d, and f the pattern for RCP4.5 without fire. 

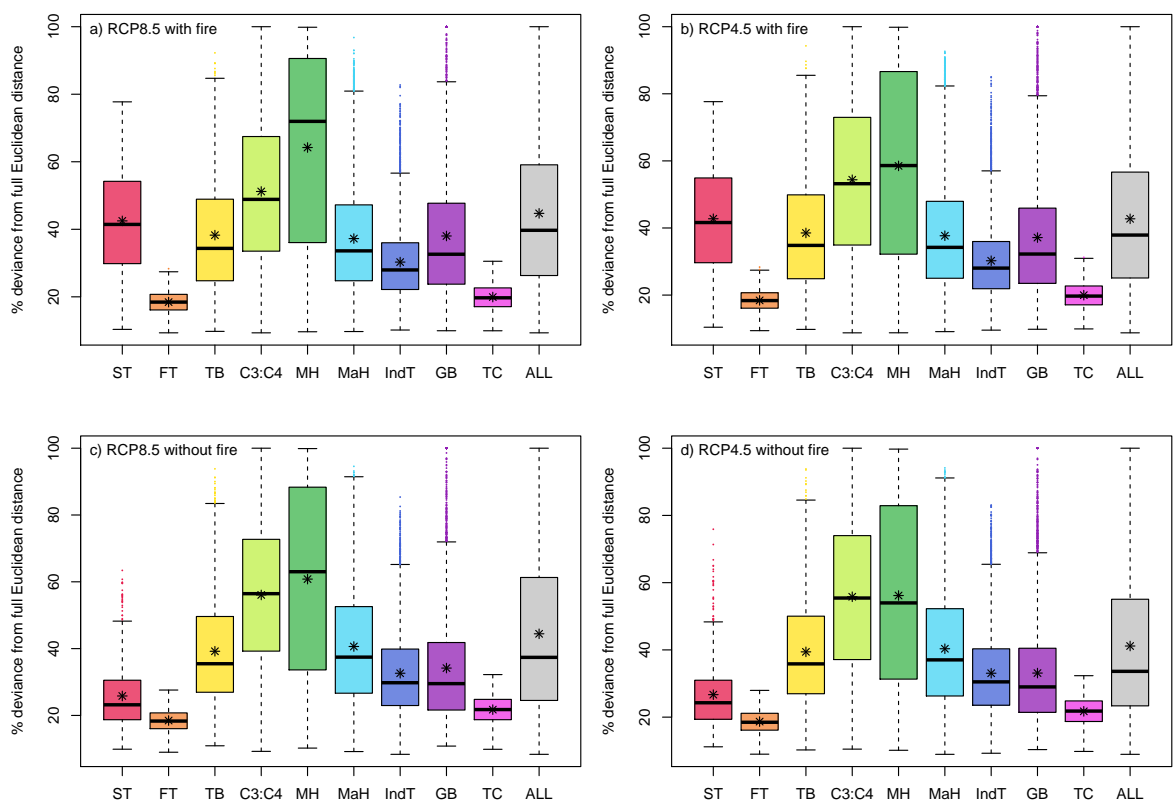

Figure S6. Percent deviance from the full Euclidean distance caused by the dominant variable, for SDPs, by identity of most influential variable (colored boxes) and across all variables irrespective of identity (grey box). ST: savanna tree cover; FT: forest tree cover; TB: aboveground tree biomass; $\mathrm{C}_{3}: \mathrm{C}_{4}: \mathrm{C}_{3}$ to $\mathrm{C}_{4}$ grass ratio; $\mathrm{MH}$ : mean tree height; $\mathrm{MaH}$ : maximum tree height; IndT: number of tree individuals; GB: aboveground grass biomass; TC: total tree cover; ALL: all variables combined; Boxes indicate first and third quartiles, the black line the median, the black star the mean value, and whiskers extend to 1.5 times the interquartile range. Percentage values of dominant variables were combined across all decades. 

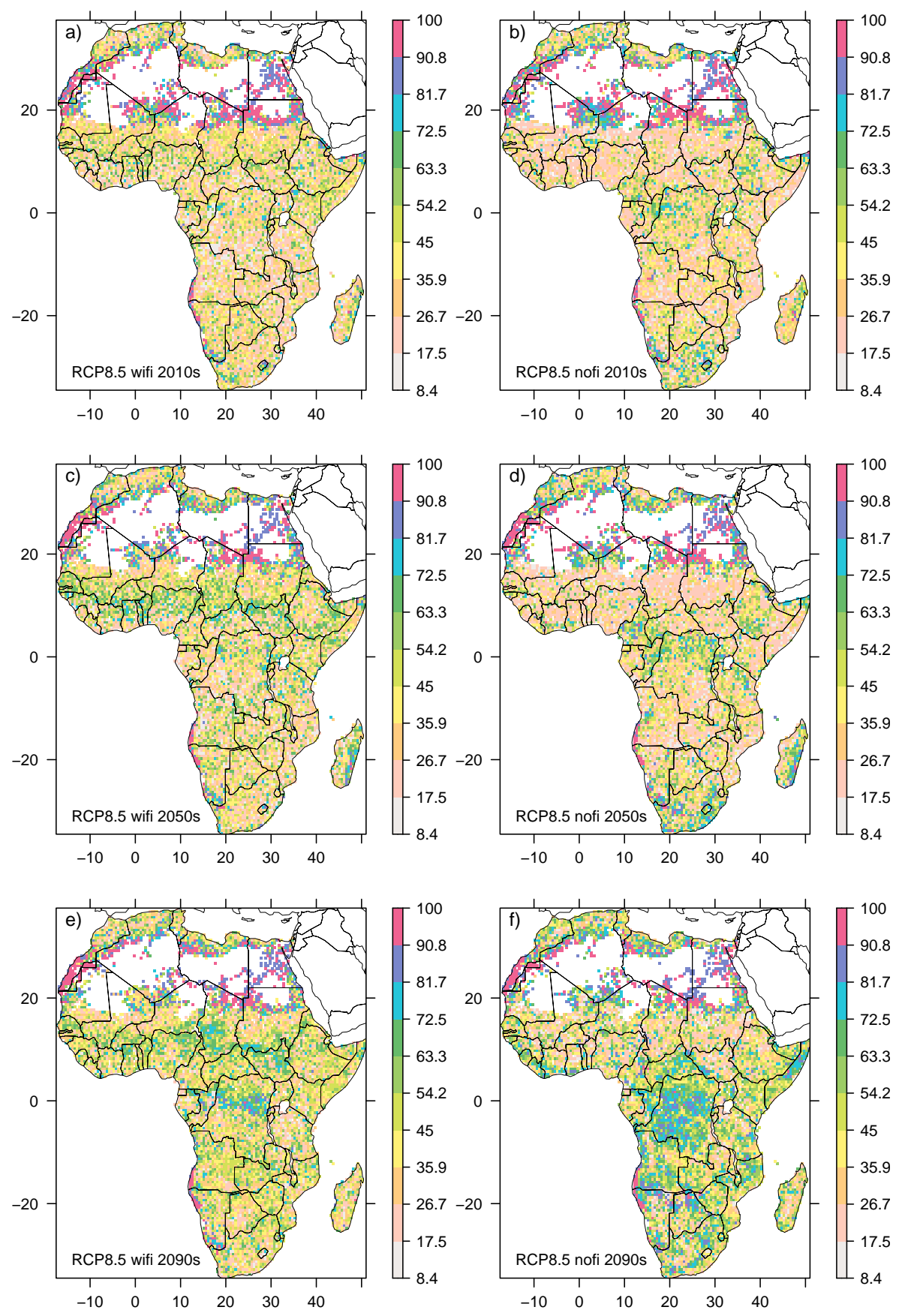

Figure S7. Spatial pattern of percent deviance from full Euclidean distance caused by the dominant variable for RCP8.5, for SDPs. Panels a, $\mathrm{c}$ and $\mathrm{e}$ are for SDPs from simulations including fire, panels $\mathrm{b}, \mathrm{d}$ and $\mathrm{f}$ for SDPs from simulations without fire. 

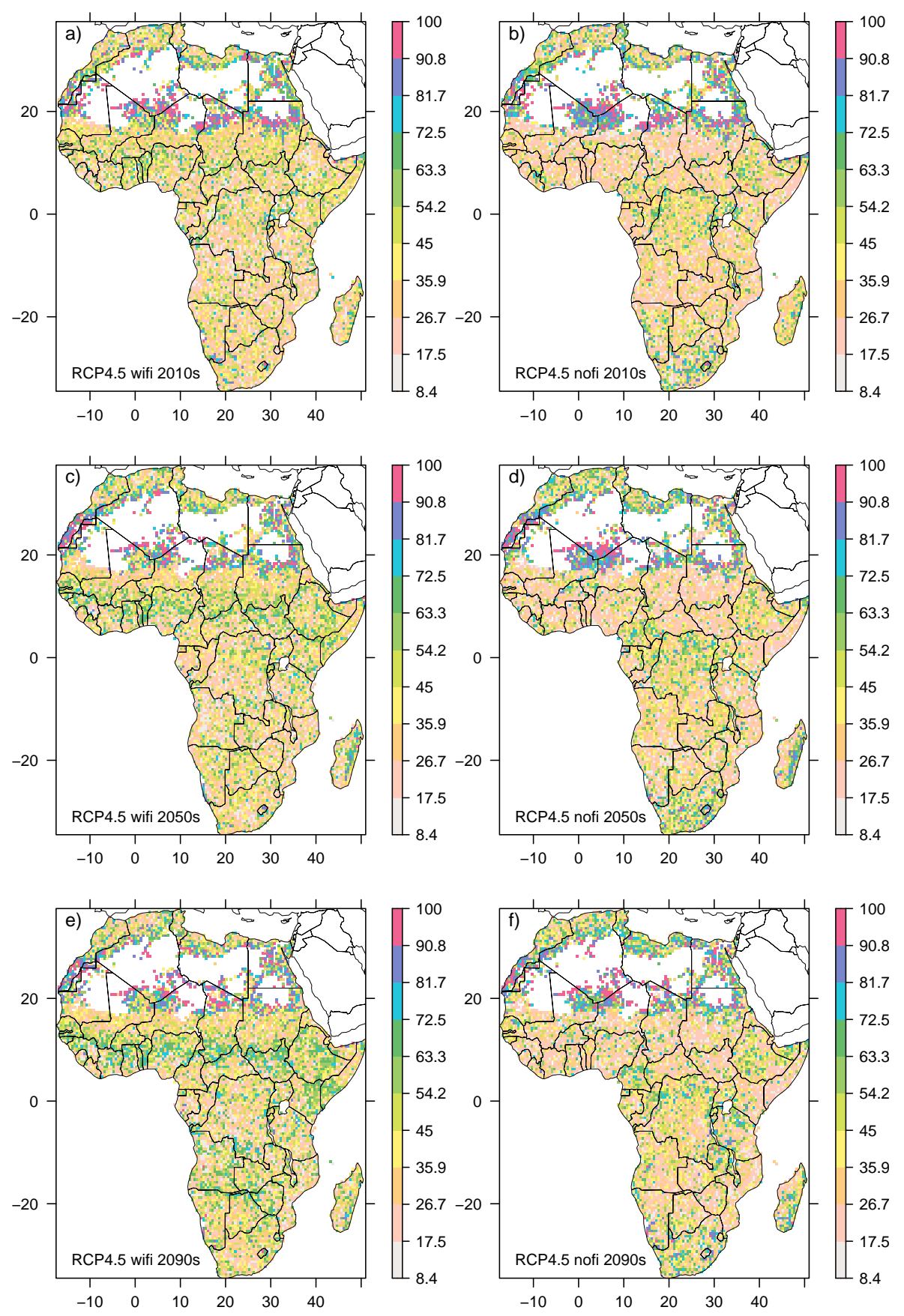

Figure S8. Spatial pattern of percent deviance from full Euclidean distance caused by the dominant variable for RCP4.5, for SDPs. Panels a, $\mathrm{c}$ and $\mathrm{e}$ are for SDPs from simulations including fire, panels $\mathrm{b}, \mathrm{d}$ and $\mathrm{f}$ for SDPs from simulations without fire. 

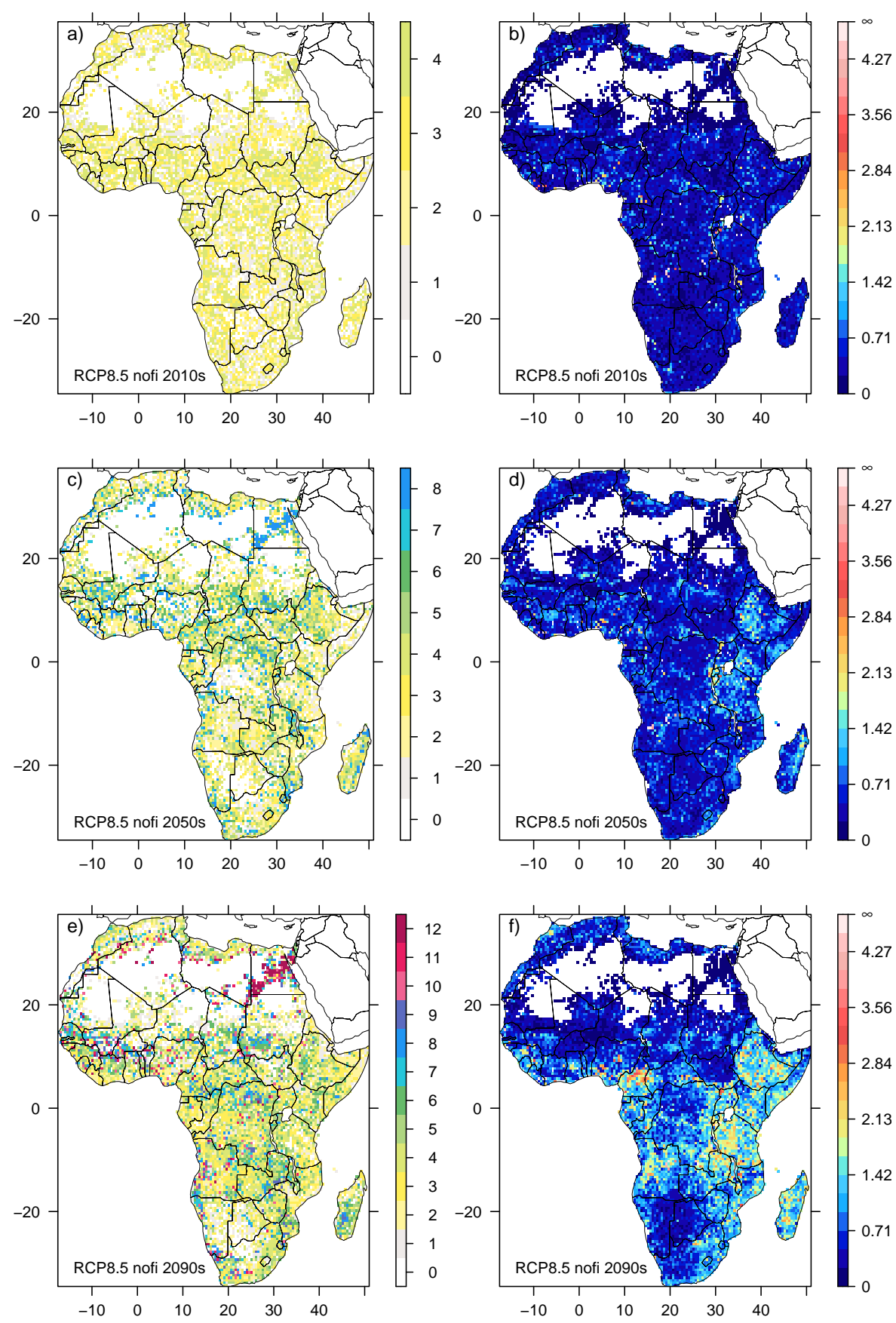

Figure S9. Spatial patterns of lag time between CDPs for RCP8.5 without fire (panels a, c and e), and residual Euclidean distance between CDPs (panels b, d and f), for three selected decades (2010-2019, 2050-2059, 2090-2099). 

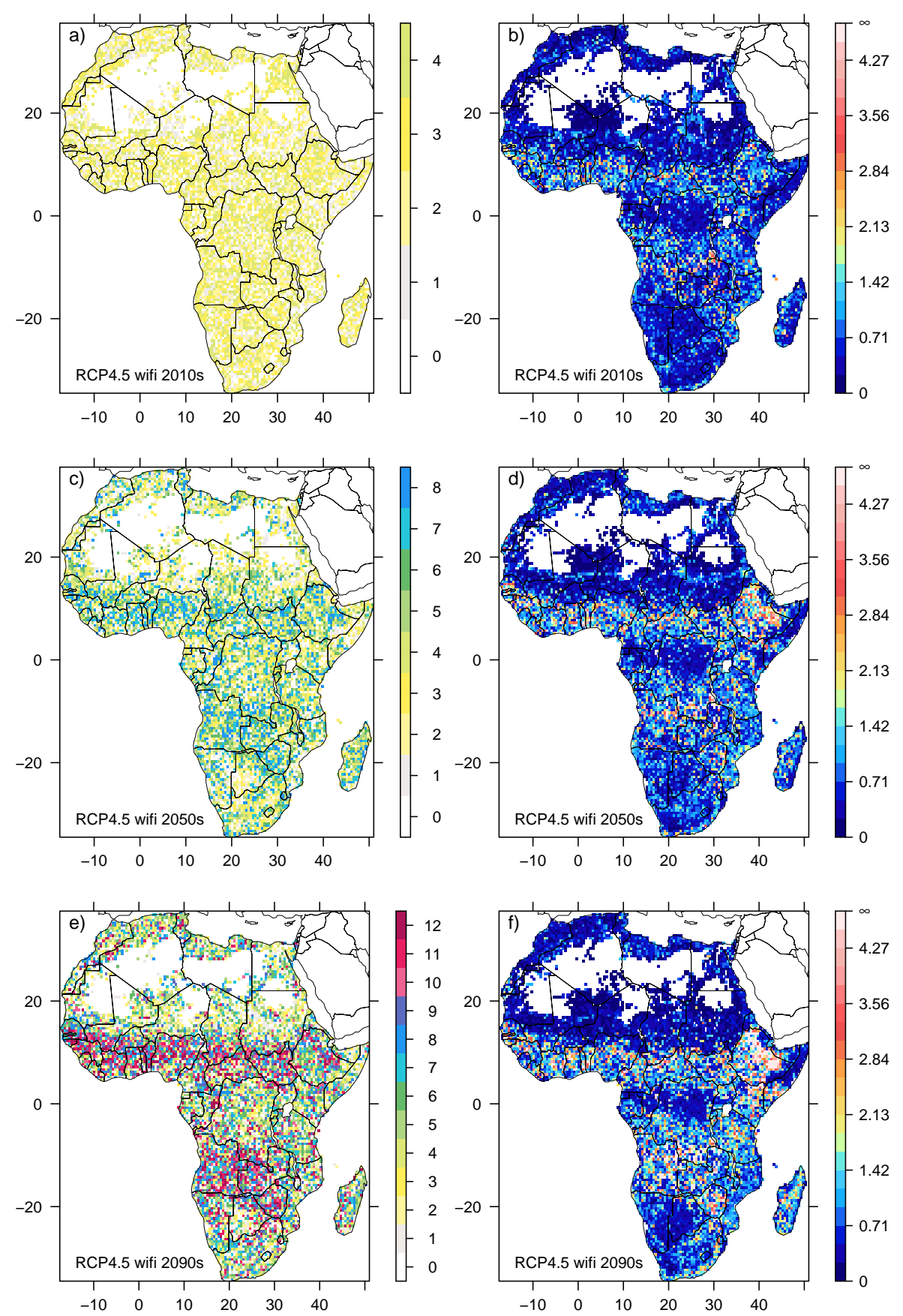

Figure S10. Spatial patterns of lag time between CDPs for RCP4.5 with fire (panels a, c and e), and residual Euclidean distance between CDPs (panels b, d and f), for three selected decades (2010-2019, 2050-2059, 2090-2099). 

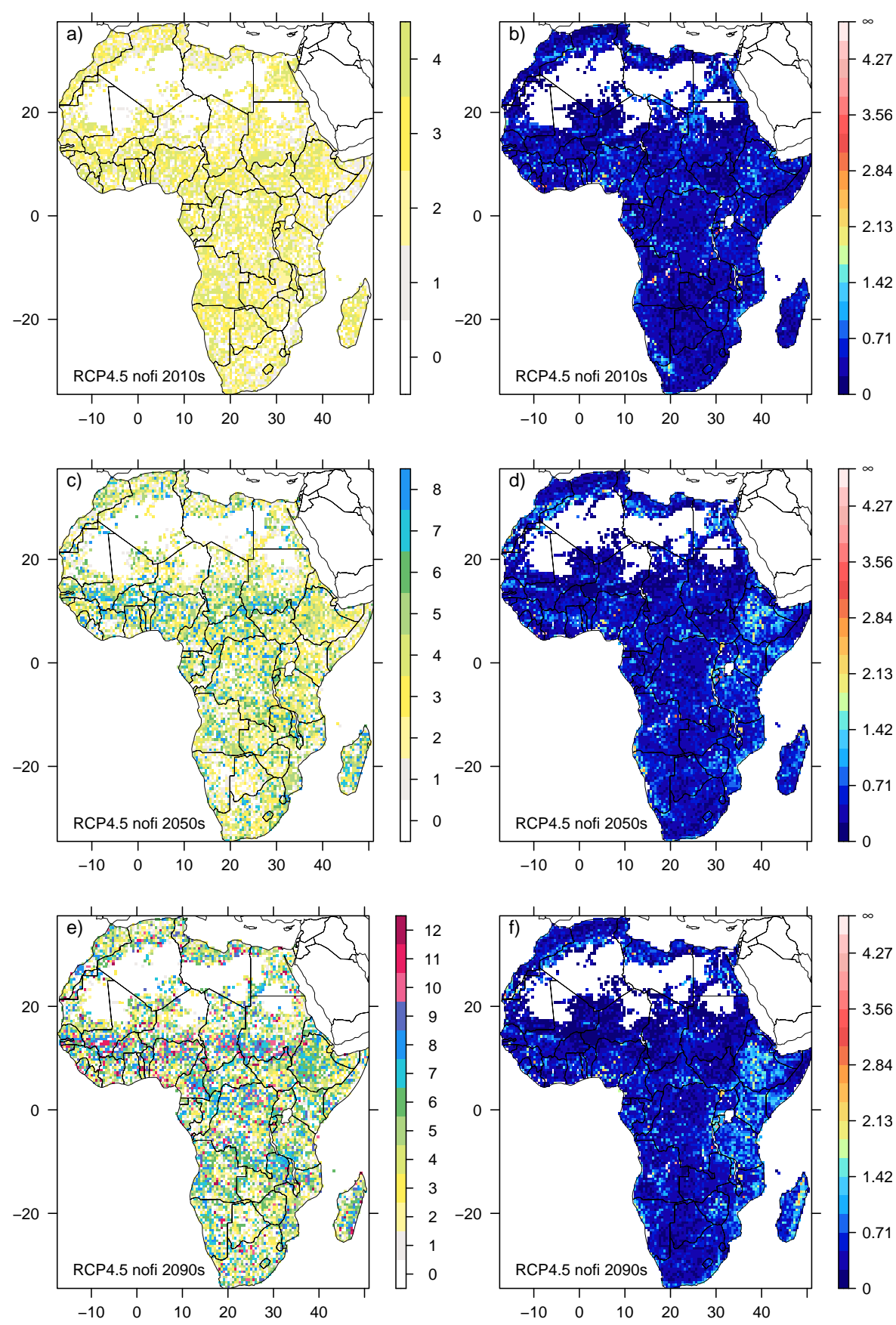

Figure S11. Spatial patterns of lag time between CDPs for RCP4.5 without fire (panels a, c and e), and residual Euclidean distance between CDPs (panels b, d and e), for three selected decades (2010-2019, 2050-2059, 2090-2099). 

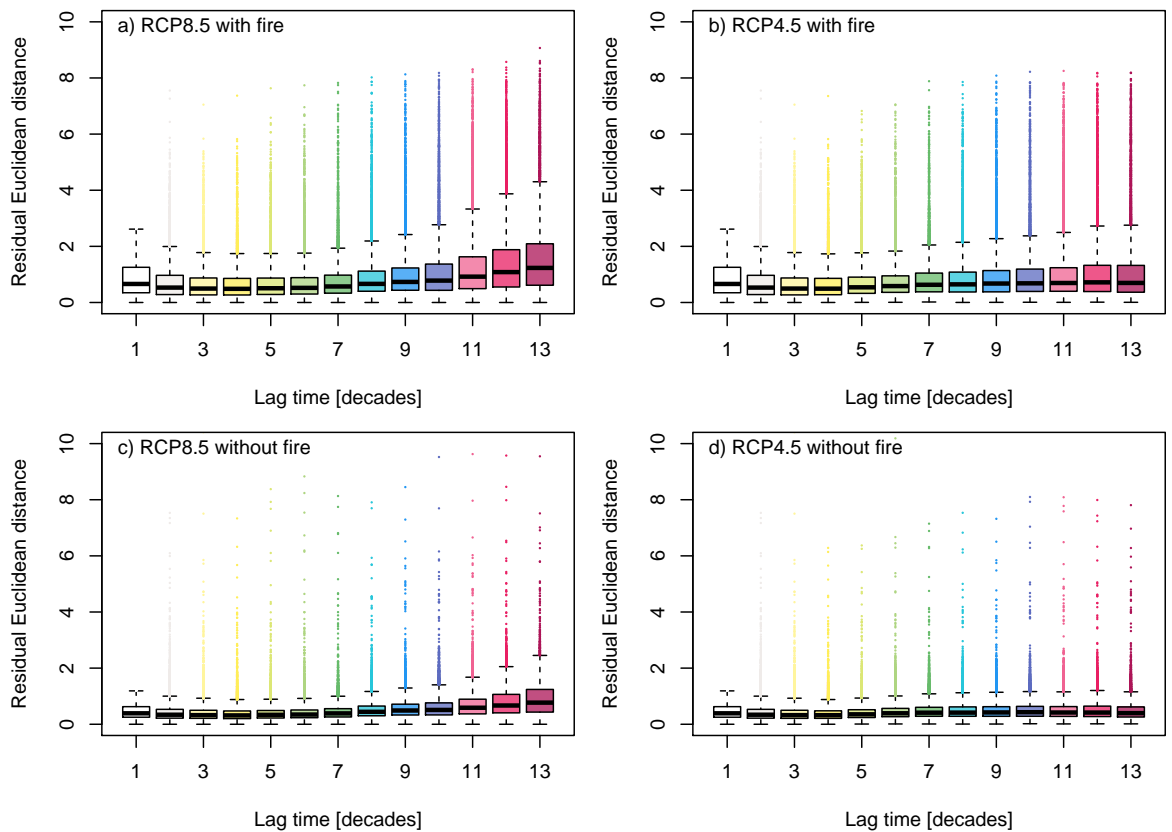

Figure S12. Residual distance between CDPs shown against lag time, irrespective of the transient decade in which these pairs occur. Boxes indicate first and third quartiles, the black line the median, and whiskers extend to 1.5 times the interquartile range. 

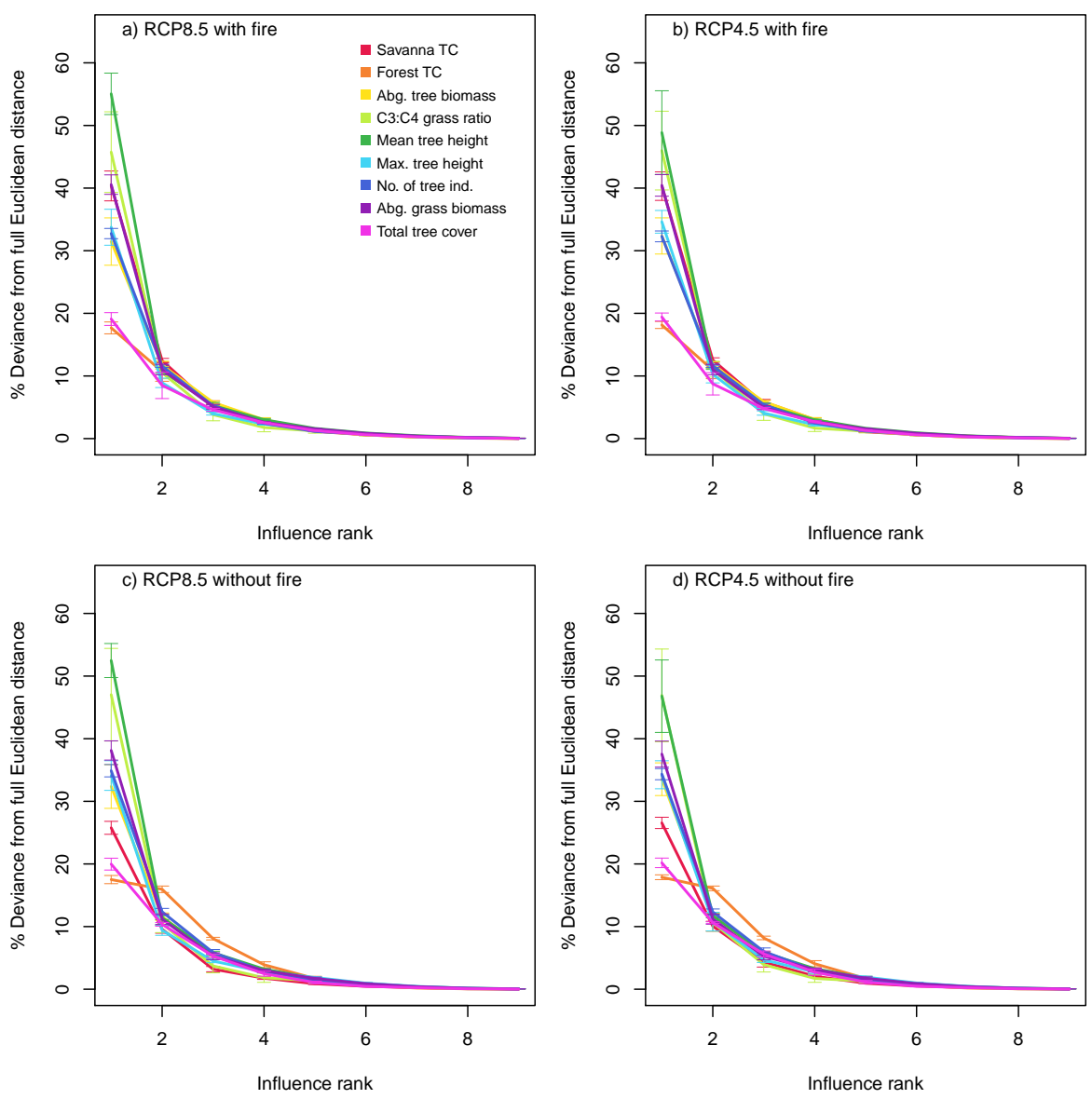

Figure S13. Decay of influence on full Euclidean distance between CDPs according to variable rank, and separated by variable identity. The second-most influential variable had substantially less influence on full Euclidean distance than the dominant variable, and influence of higher-ranked variables rapidly declined. 

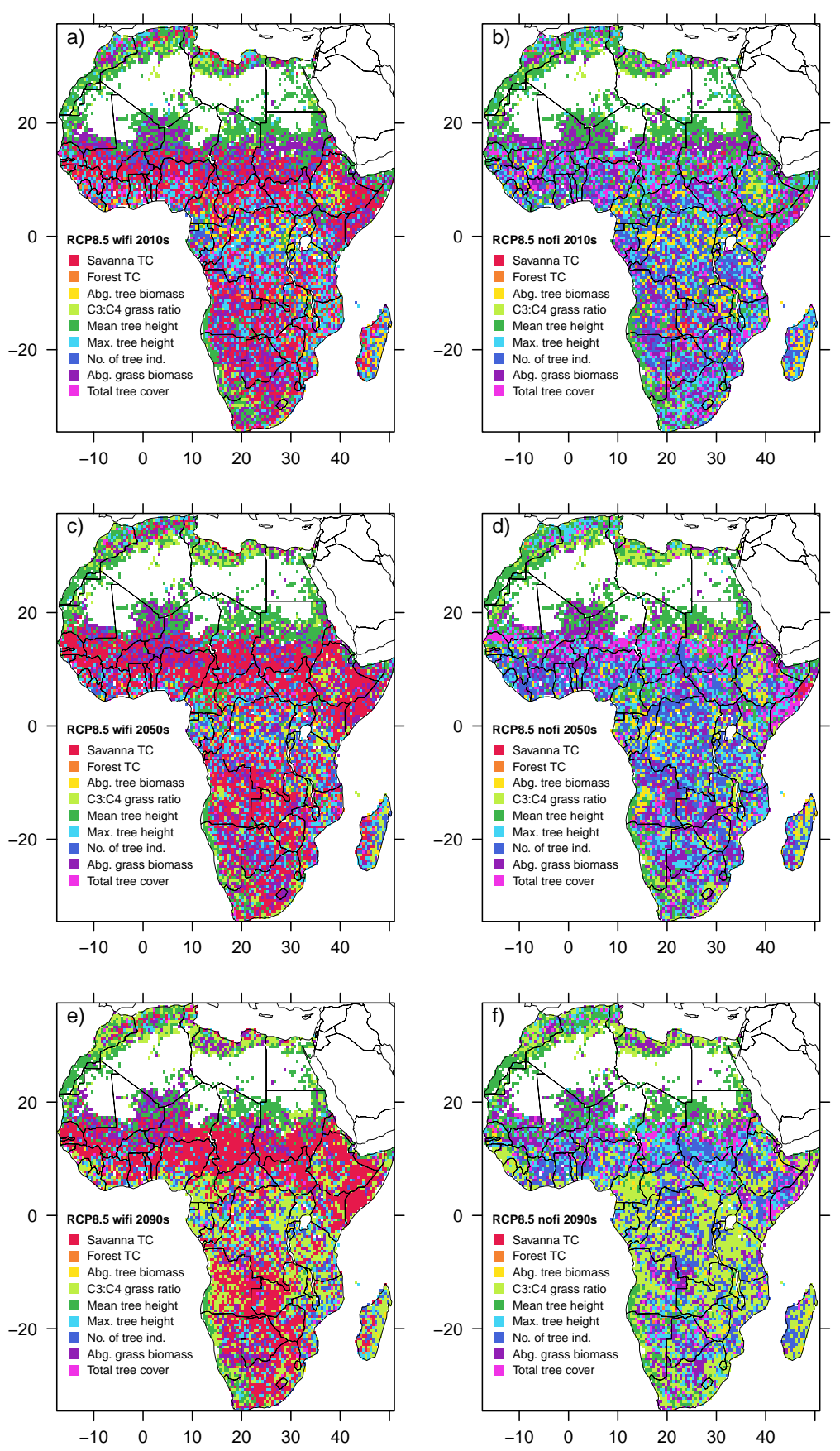

Figure S14. Spatial pattern of dominant variables with respect to Euclidean distance between CDPs for RCP8.5 for the three reference decades. Panels a, $\mathrm{c}$ and e show the spatial pattern for RCP8.5 with fire, panels b, $\mathrm{d}$ and $\mathrm{f}$ the pattern for RCP8.5 without fire. 

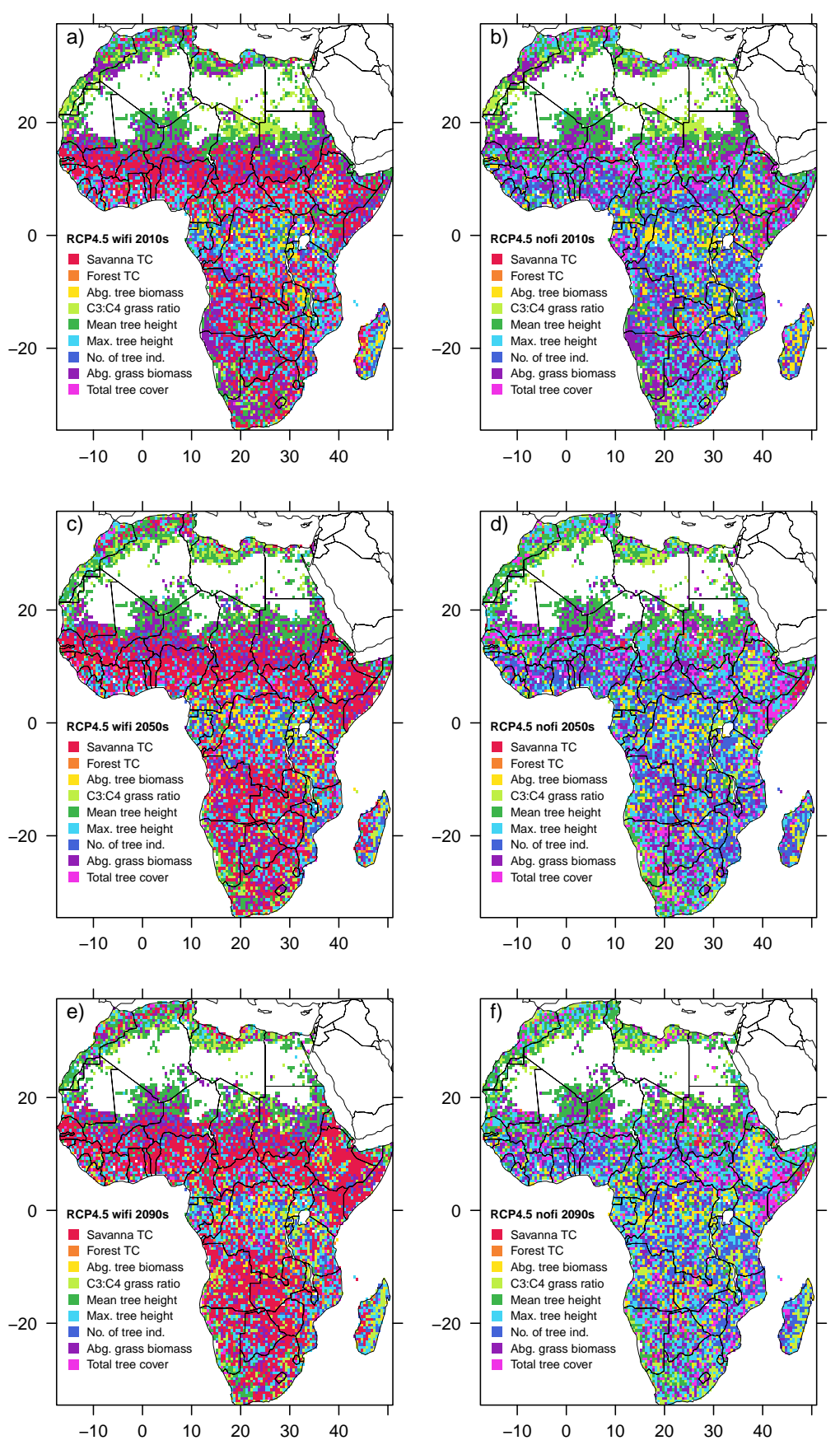

Figure S15. Spatial pattern of dominant variables with respect to Euclidean distance between CDPs for RCP4.5 for the three reference decades. Panels a, $\mathrm{c}$ and e show the spatial pattern for RCP4.5 with fire, panels b, $\mathrm{d}$ and $\mathrm{f}$ the pattern for RCP4.5 without fire. 

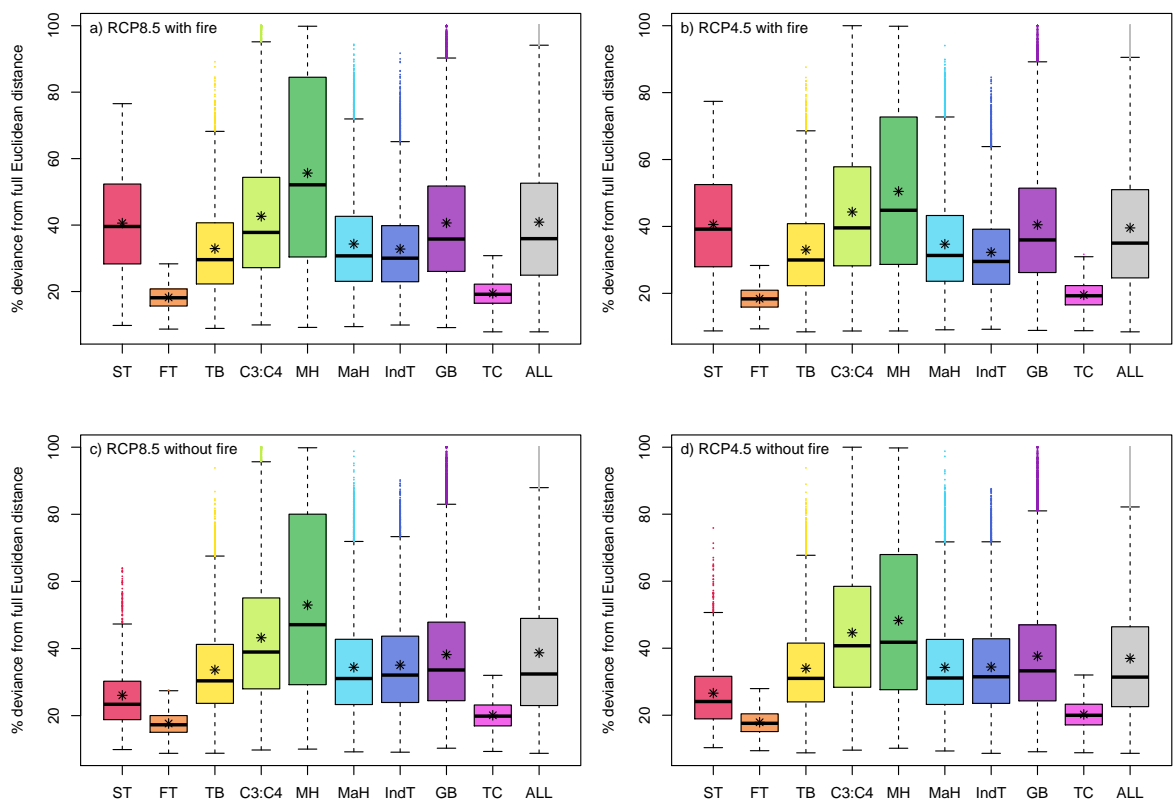

Figure S16. Percent deviance from the full Euclidean distance caused by the dominant variable, for CDPs, by identity of most influential variable (colored boxes) and across all variables irrespective of identity (grey box). ST: savanna tree cover; FT: forest tree cover; TB: aboveground tree biomass; $\mathrm{C}_{3}: \mathrm{C}_{4}: \mathrm{C}_{3}$ to $\mathrm{C}_{4}$ grass ratio; $\mathrm{MH}$ : mean tree height; MaH: maximum tree height; IndT: number of tree individuals; GB: aboveground grass biomass; TC: total tree cover; ALL: all variables combined; Boxes indicate first and third quartiles, the black line the median, the black star the mean value, and whiskers extend to 1.5 times the interquartile range. Percentage values of dominant variables were combined across all decades. 

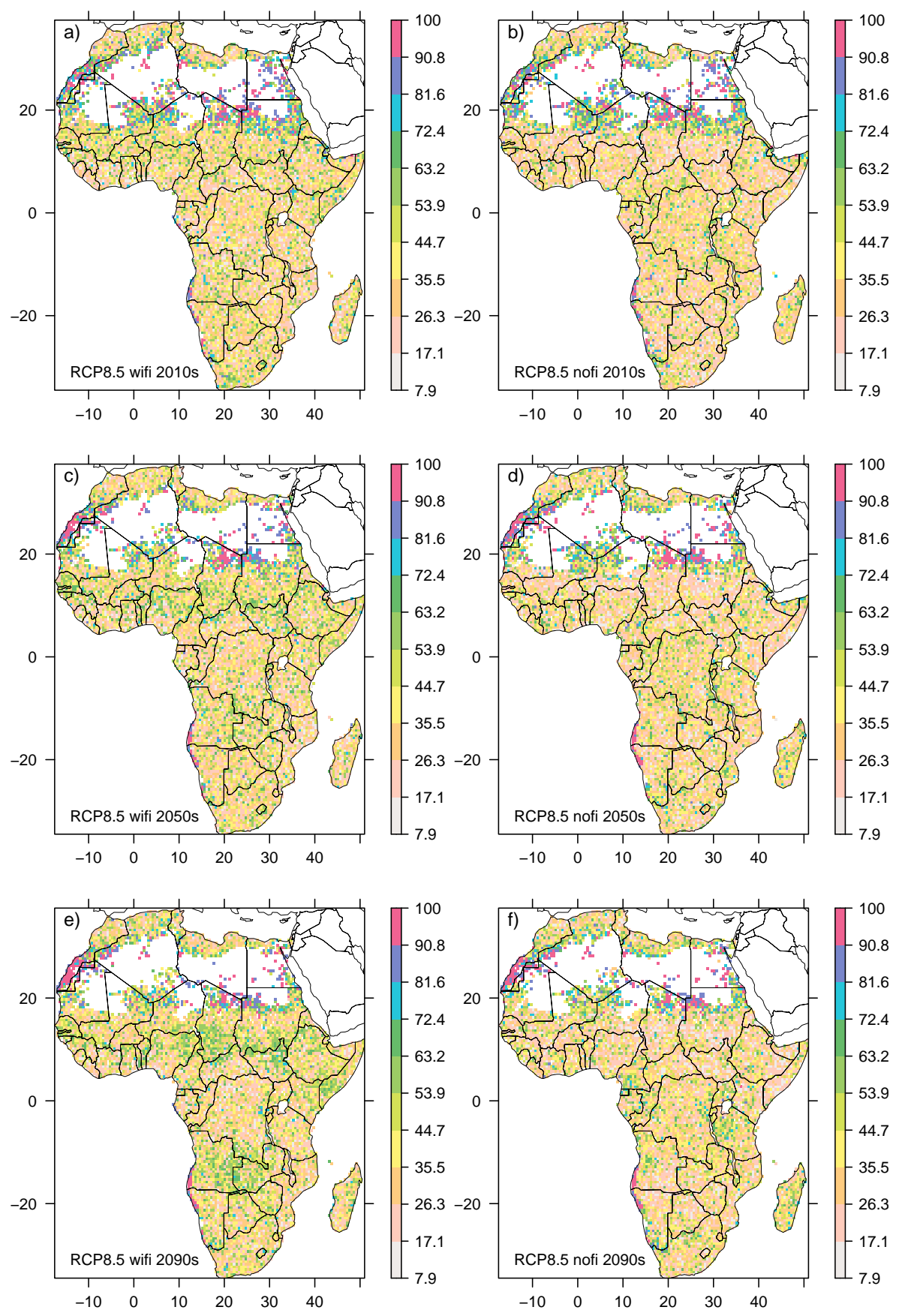

Figure S17. Spatial pattern of percent deviance from full Euclidean distance caused by the dominant variable for RCP8.5, for CDPs. Panels $\mathrm{a}, \mathrm{c}$ and e refer to CDPs from simulations including fire, panels $\mathrm{b}, \mathrm{d}$ and $\mathrm{f}$ to CDPs from simulations without fire. 

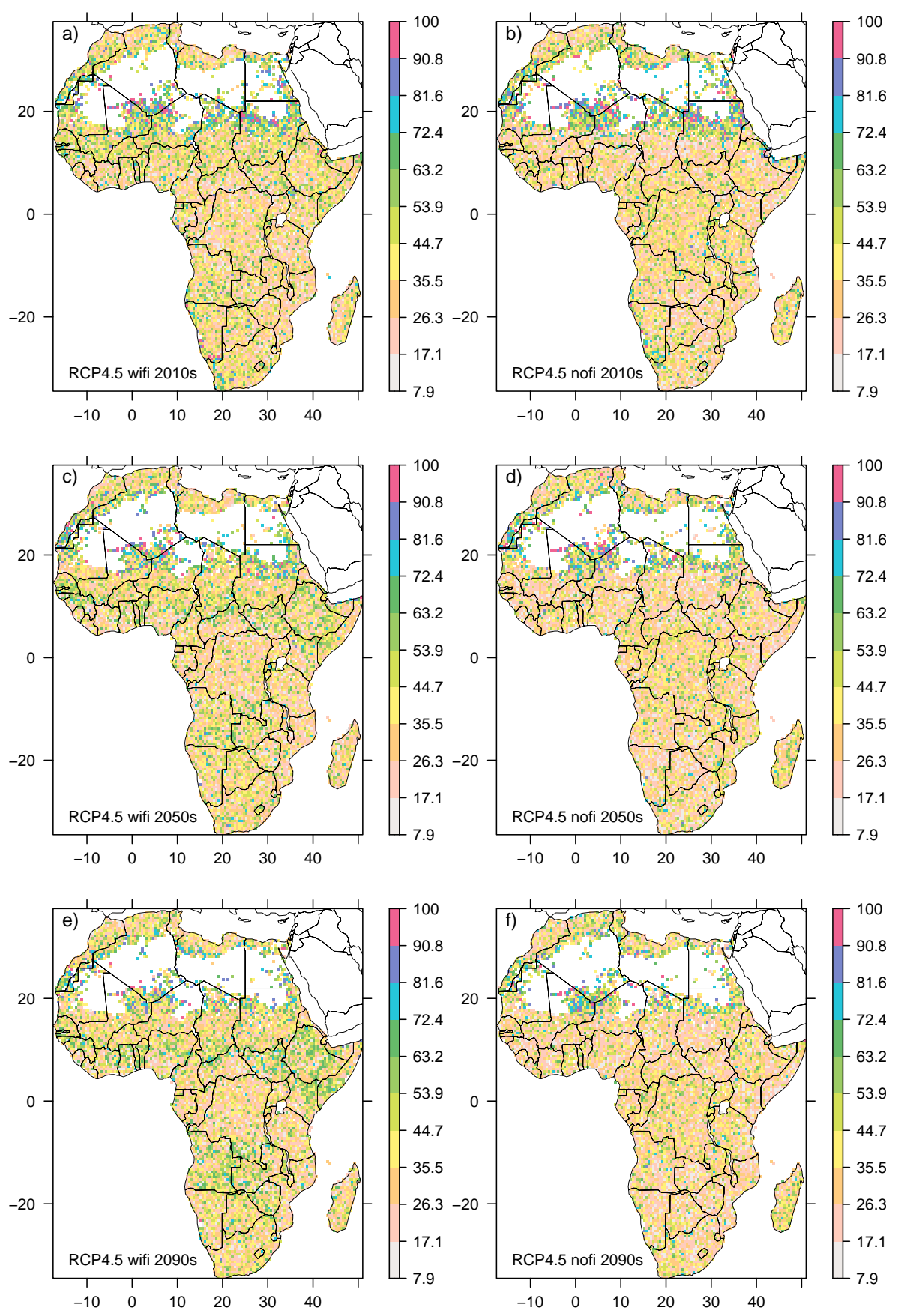

Figure S18. Spatial pattern of percent deviance from full Euclidean distance caused by the dominant variable for RCP4.5, for CDPs. Panels $\mathrm{a}, \mathrm{c}$ and e refer to CDPs from simulations including fire, panels $\mathrm{b}, \mathrm{d}$ and $\mathrm{f}$ to CDPs from simulations without fire. 

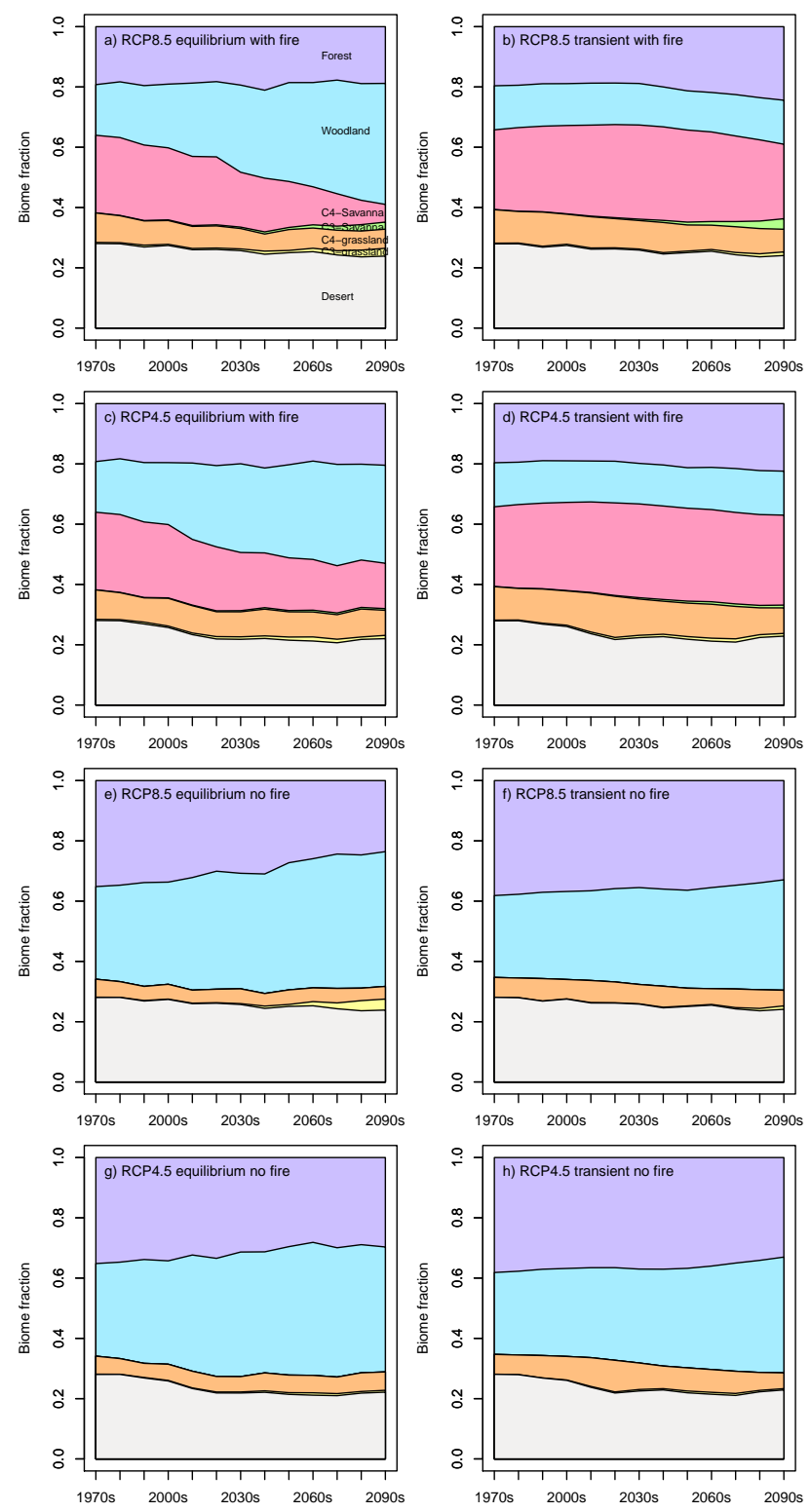

Figure S19. Fractions of African area covered by specific biome types, as time series stacks. Panels a-d show biome cover fractions for simulations with fire, panels e-h show biome cover fractions for simulations without fire. 

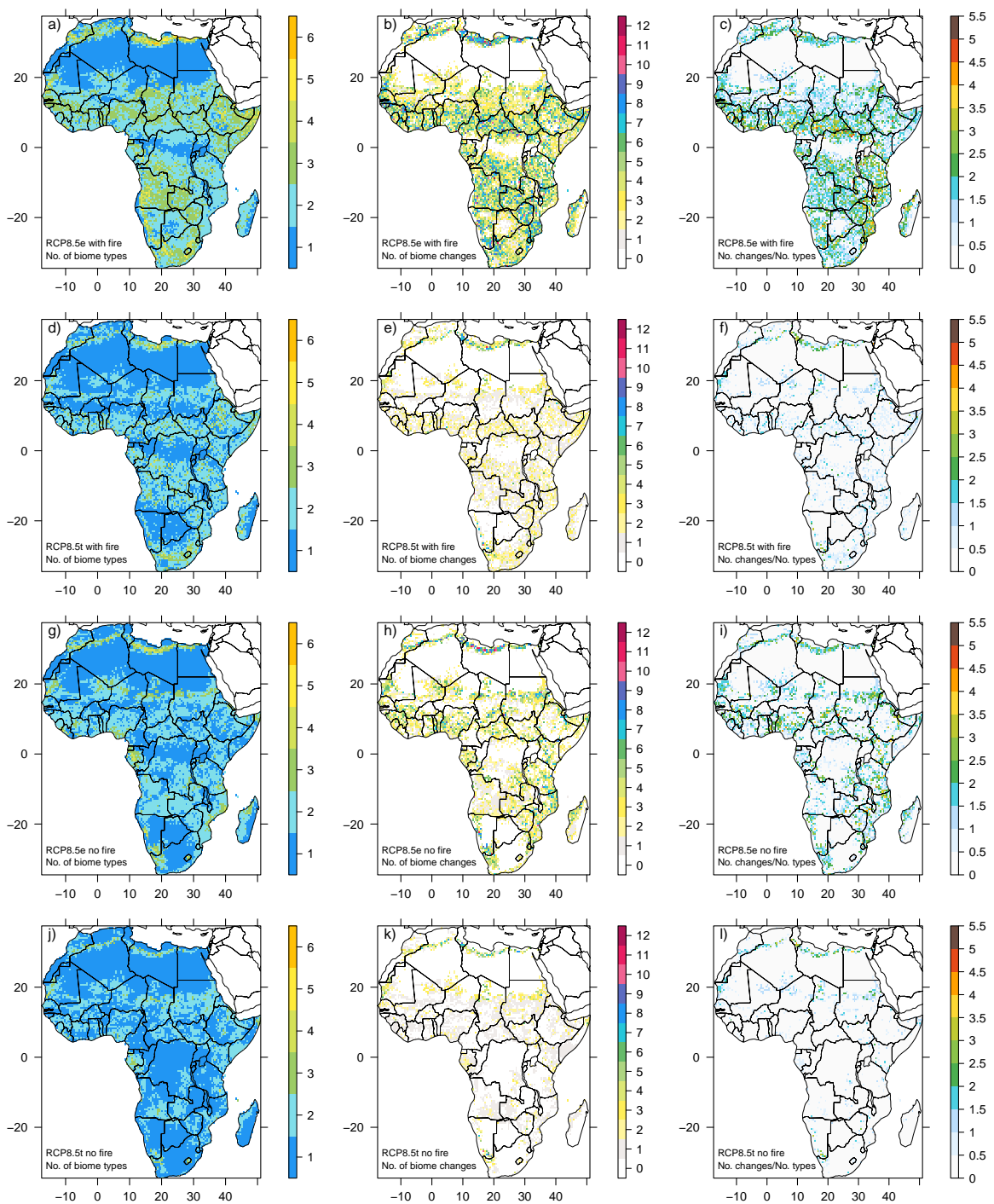

Figure S20. Overview showing the number of biome types assumed by a given grid cell (left column), the number of biome changes experienced by a given grid cell over the course of 13 decades (central column), and the ratio between number of biome changes experienced by a given grid cell and the number of biome types assumed by that grid cell (right column), for RCP8.5 simulation scenarios. A large ratio between number of biome changes and number of biome types assumed indicates a grid cell is flickering back and forth between two or more biome types, i.e., the transition is less definite than at a low ratio. 

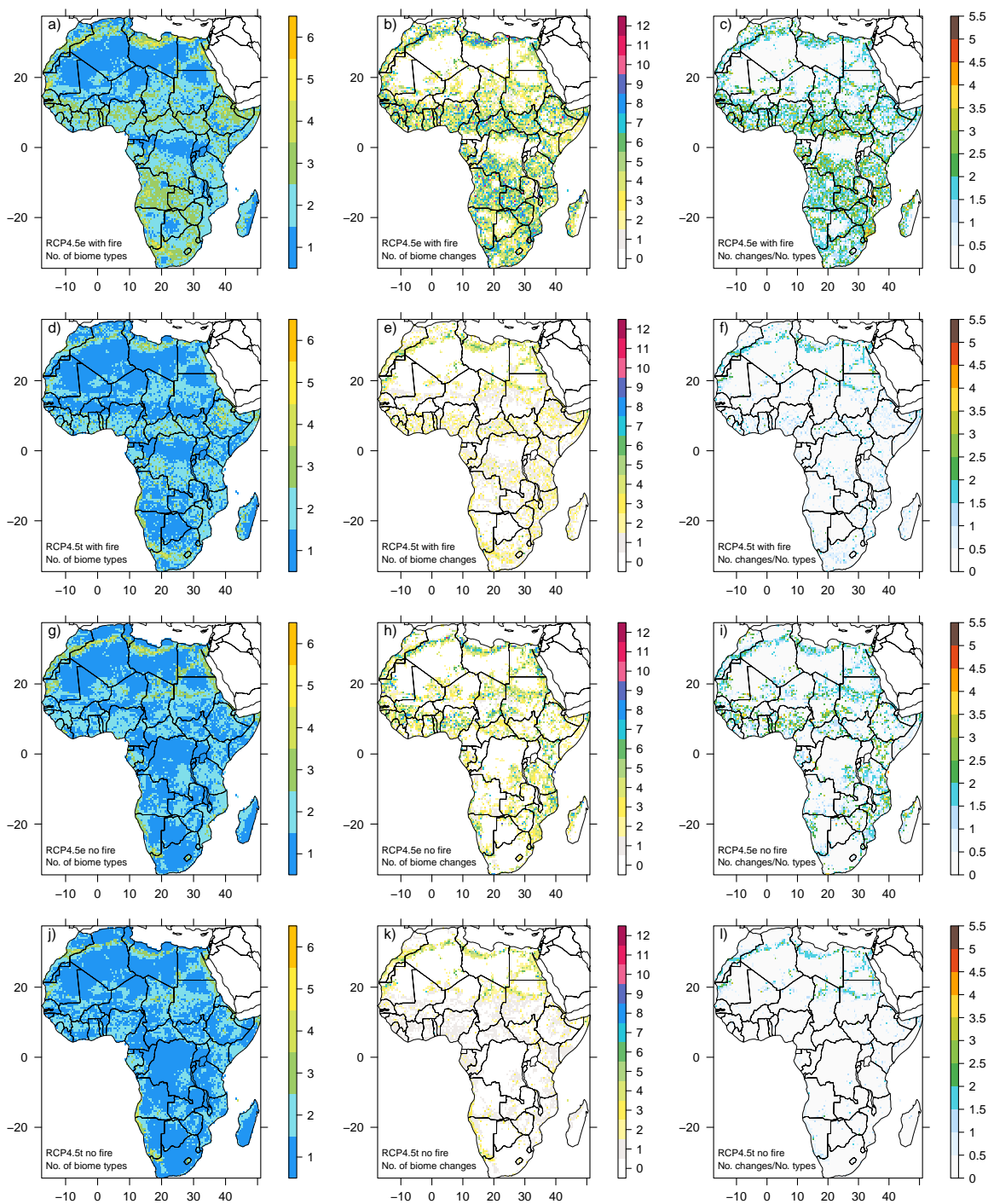

Figure S21. Overview showing the number of biome types assumed by a given grid cell (left column), the number of biome changes experienced by a given grid cell over the course of 13 decades (central column), and the ratio between number of biome changes experienced by a given grid cell and the number of biome types assumed by that grid cell (right column), for RCP4.5 simulation scenarios. A large ratio between number of biome changes and number of biome types assumed indicates a grid cell is flickering back and forth between two or more biome types, i.e., the transition is less definite than at a low ratio. 


\section{SUPPLEMENT B}

Additional supplementary figures illustrating temporal changes of biome states, as well as differences between biome states of

5 different scenarios.

\section{Time series comparison}

Figure series of Sankey diagrams that show biome change between the 2010s and 2050s, and between 2050s and 2090s, within a given scenario.

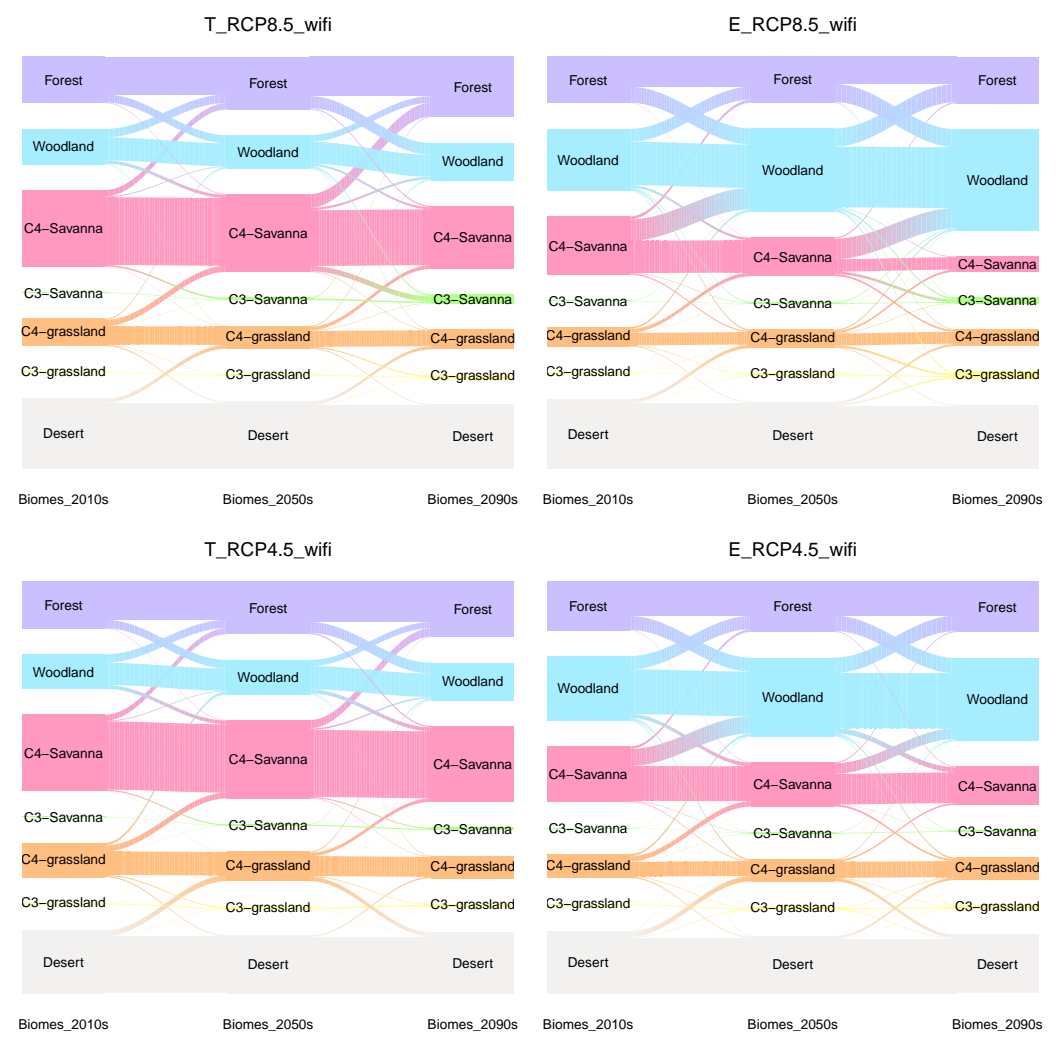

Figure B1. Sankey diagrams showing the change of biomes between the three decades of interest, for both RCP scenarios with fire. "T_" denotes transient scenarios, "E_ "denotes equilibrium scenarios. 


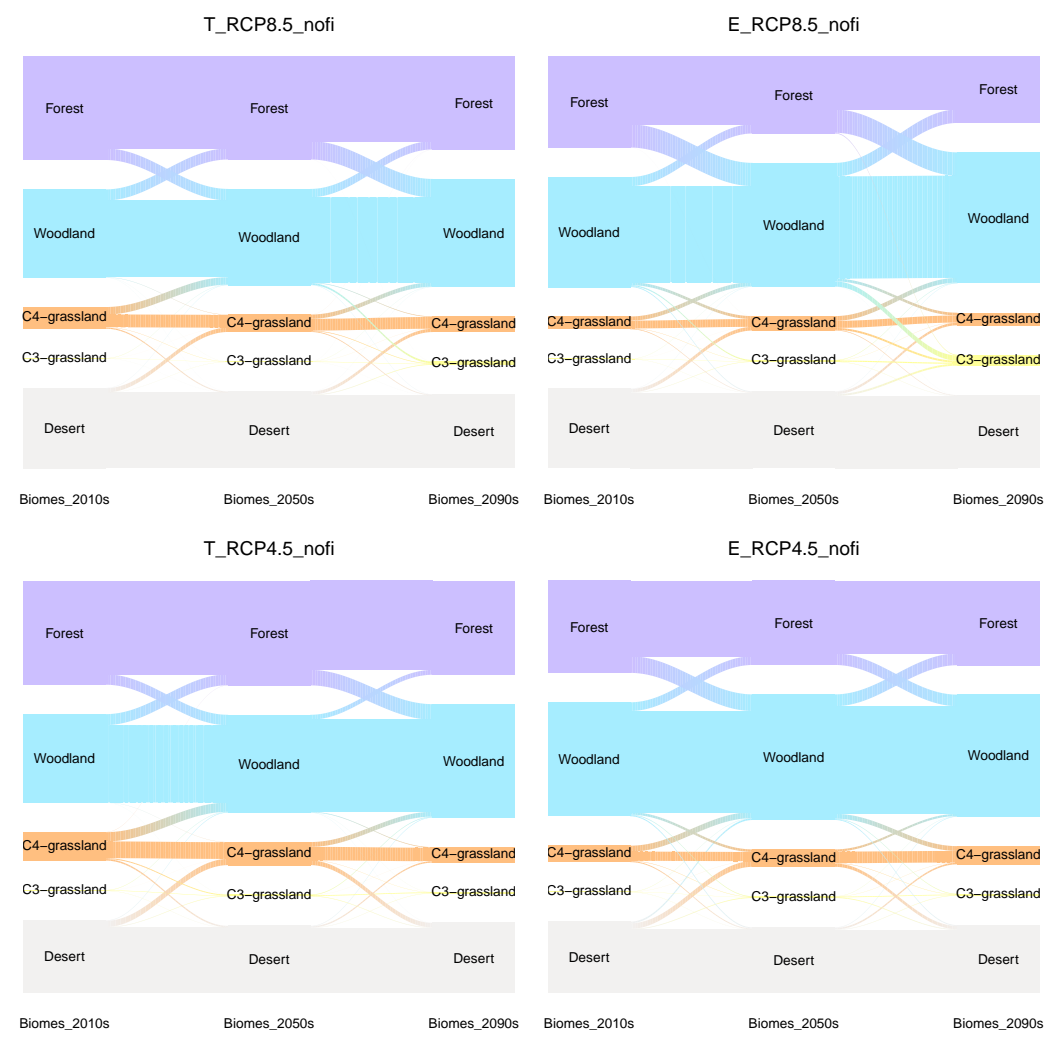

Figure B2. Sankey diagrams showing the change of biomes between the three decades of interest, for both RCP scenarios without fire. "T_ " denotes transient scenarios, "E_"denotes equilibrium scenarios. 


\section{Comparison between scenarios - Fire against no fire}

10 Sankey diagrams showing difference in biome type between scenarios with fire and scenarios without fire for the three decades of interest (2010s, 2050s, 2090s).

T 8.5 wifinofi_2010s

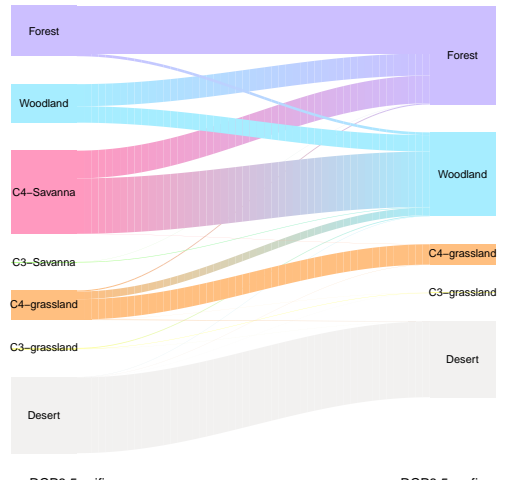

T_8.5_wifinofi_2050s
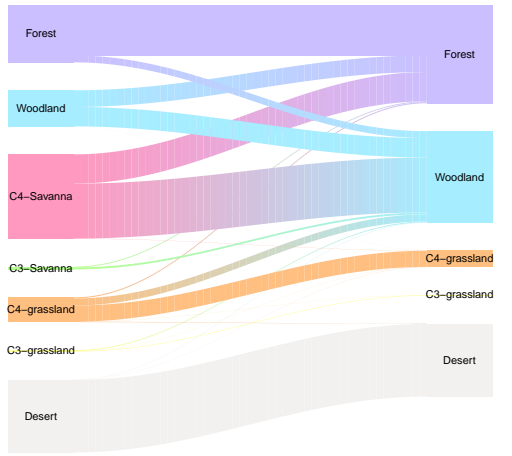

RCP8.5 wifi $\quad$ RCP8.5 nofi

T 8.5 wifinofi_ 2090 s
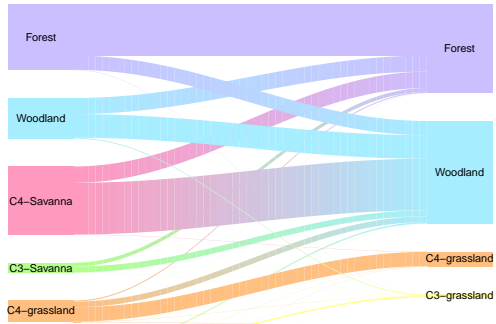

c3-grassland

Desert

RCP8.5 wifi
E 8.5 wifinofi 2010s

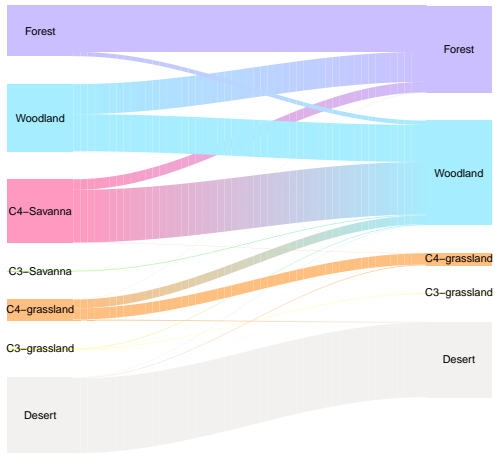

RCP8.5 wifi RCP8.5 nofi

E 8.5_wifinofi_2050s
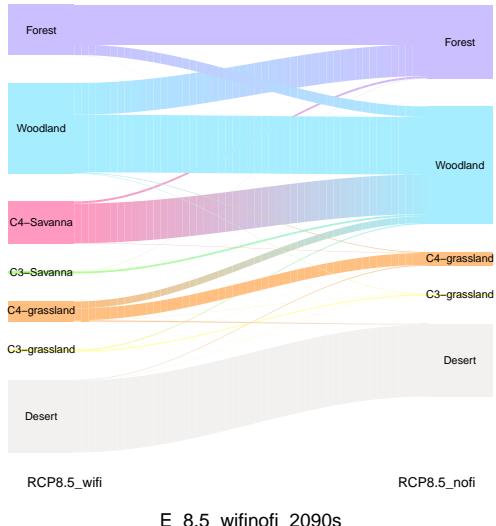

E 8.5 wifinofi 2090s
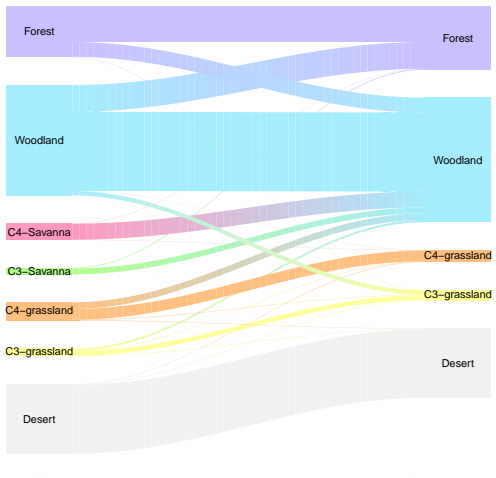

$\begin{array}{lll}\text { RCP8.5 wifi } & \text { RCP8.5 not }\end{array}$

Figure B3. Sankey diagrams showing the difference in biome type between scenarios with fire and scenarios without fire for RCP8.5, for the three decades of interest (2010s, 2050s, 2090s). ”T_"denotes transient scenarios, ”E_" denotes equilibrium scenarios. 


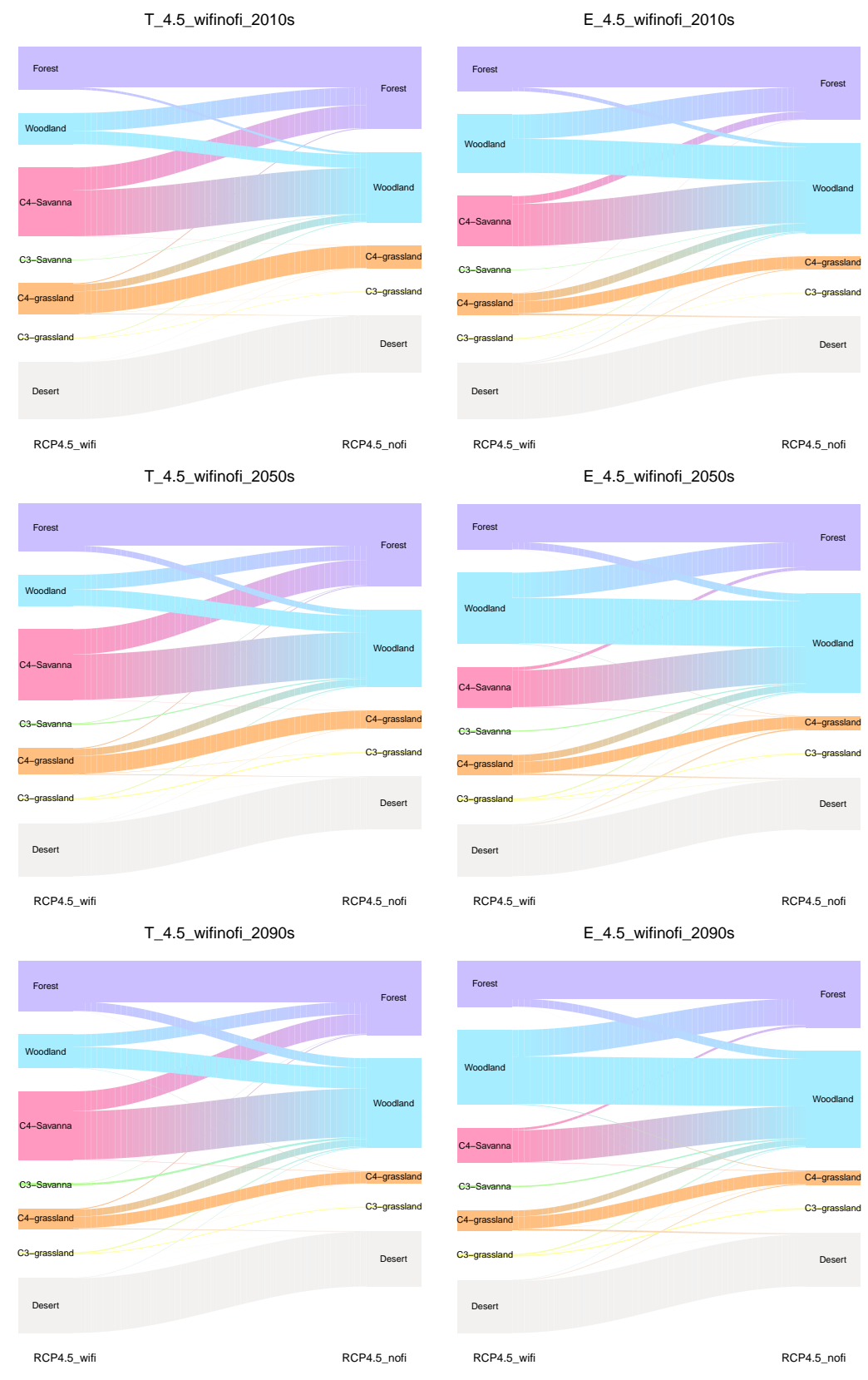

Figure B4. Sankey diagrams showing the difference in biome type between scenarios with fire and scenarios without fire for RCP4.5, for the three decades of interest (2010s, 2050s, 2090s). ”T_“denotes transient scenarios, ”E_“ denotes equilibrium scenarios. 


\section{Comparison between scenarios - Transient against equilibrium}

Sankey diagrams showing difference in biome type between transient and equilibrium scenarios for the three decades of interest (2010s, 2050s, 2090s).

8.5wifi_2010s
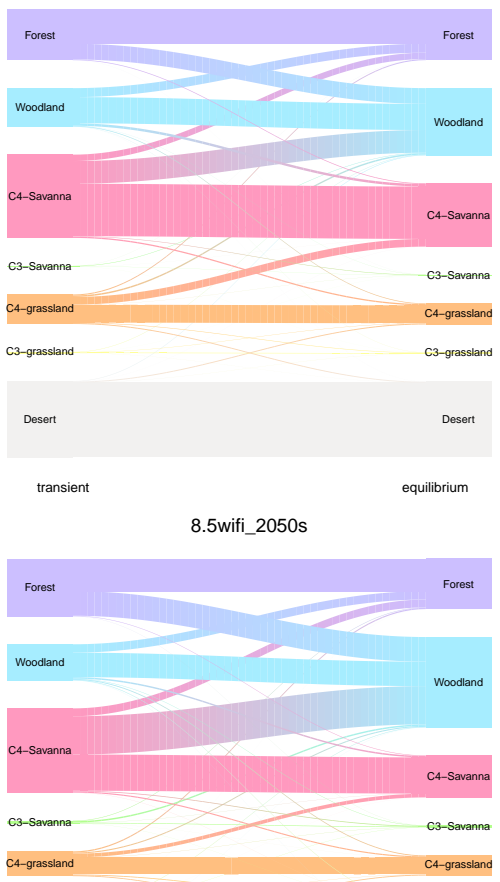

C3-grassland

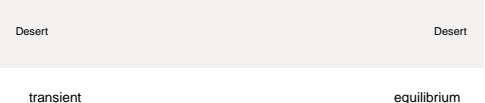

8.5wifi_2090s
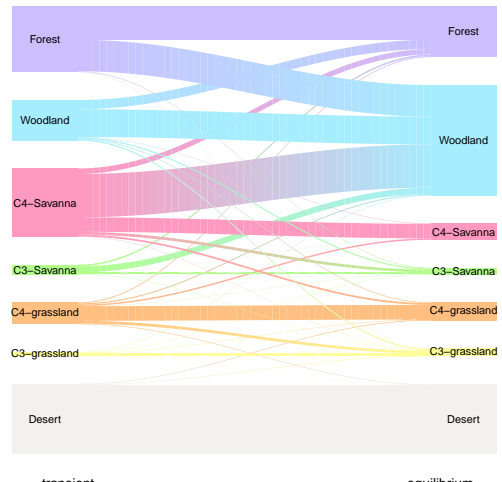

8.5nofi 2010 s
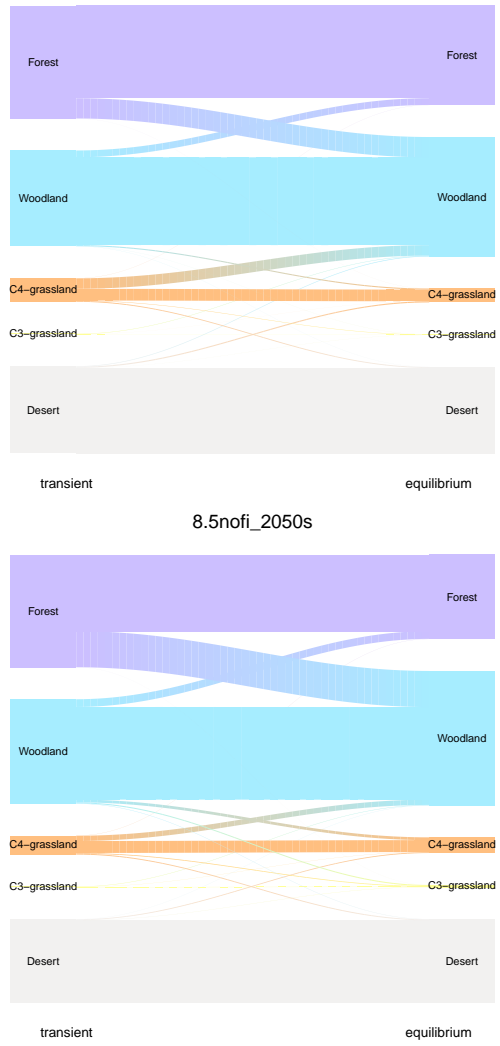

transient $\quad$ equilibrium

E_8.5-4.5_nofi_2090s
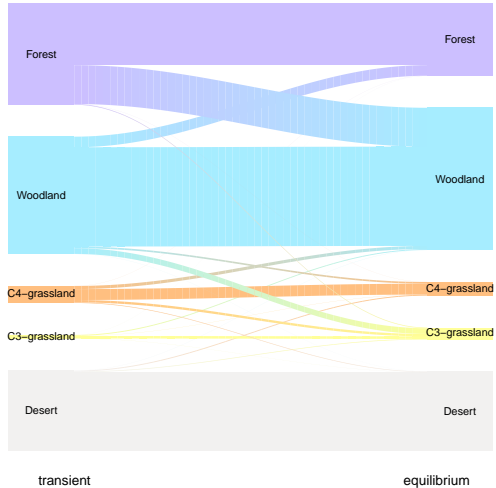

Figure B5. Sankey diagrams showing the difference in biome type between transient and equilibrium scenarios for RCP8.5, for the three decades of interest (2010s, 2050s, 2090s). "wifi“ denotes scenarios with fire, "nofi“ denotes scenarios without fire. 


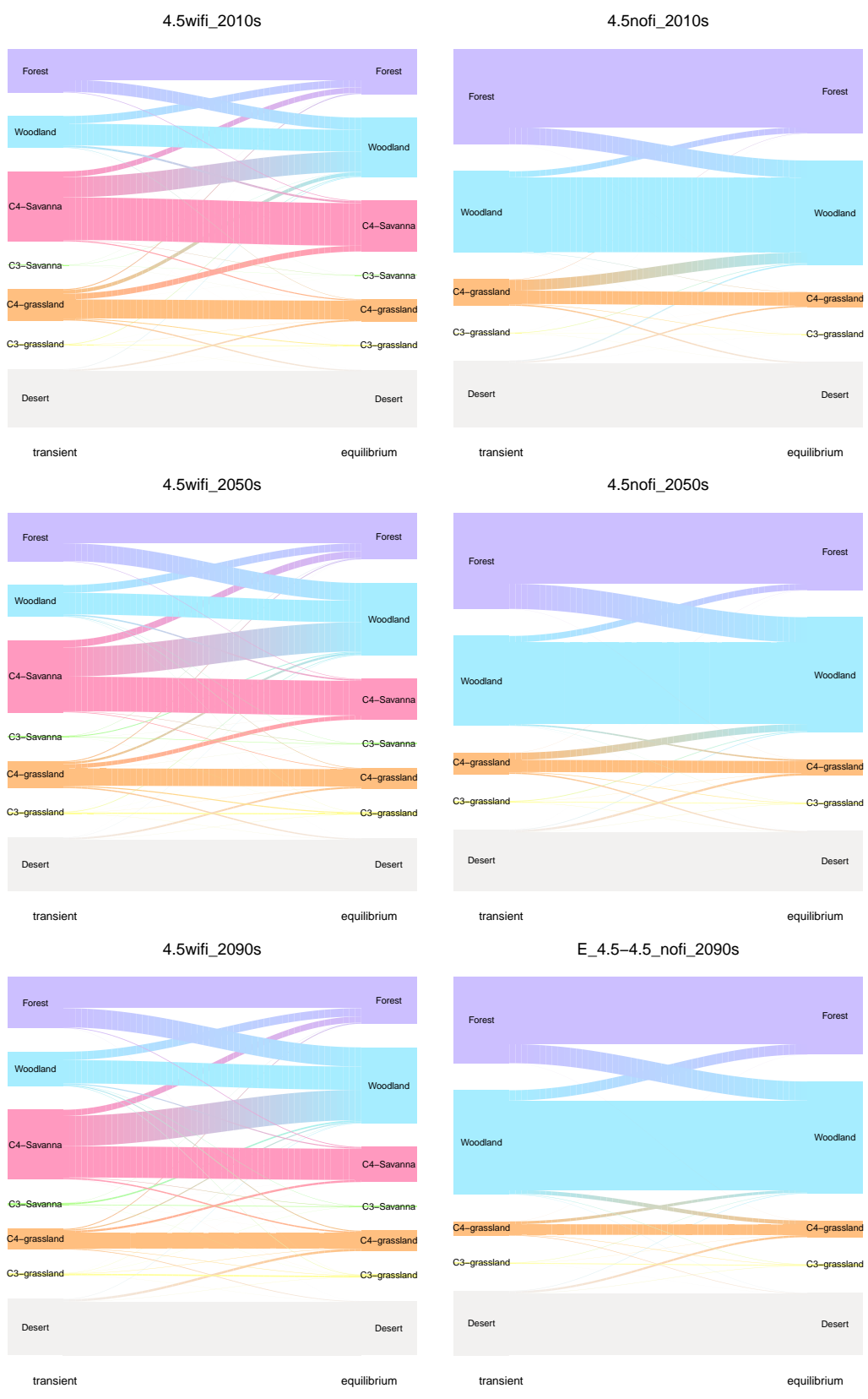

Figure B6. Sankey diagrams showing the difference in biome type between transient and equilibrium scenarios for RCP4.5, for the three decades of interest (2010s, 2050s, 2090s). "wifi“ denotes scenarios with fire, "nofi“ denotes scenarios without fire. 


\section{IV. Comparison between scenarios - RCP8.5 against RCP4.5}

Sankey diagrams showing difference in biome type between RCP8.5 and RCP4.5 for the three decades of interest (2010s, 2050s, 2090s).
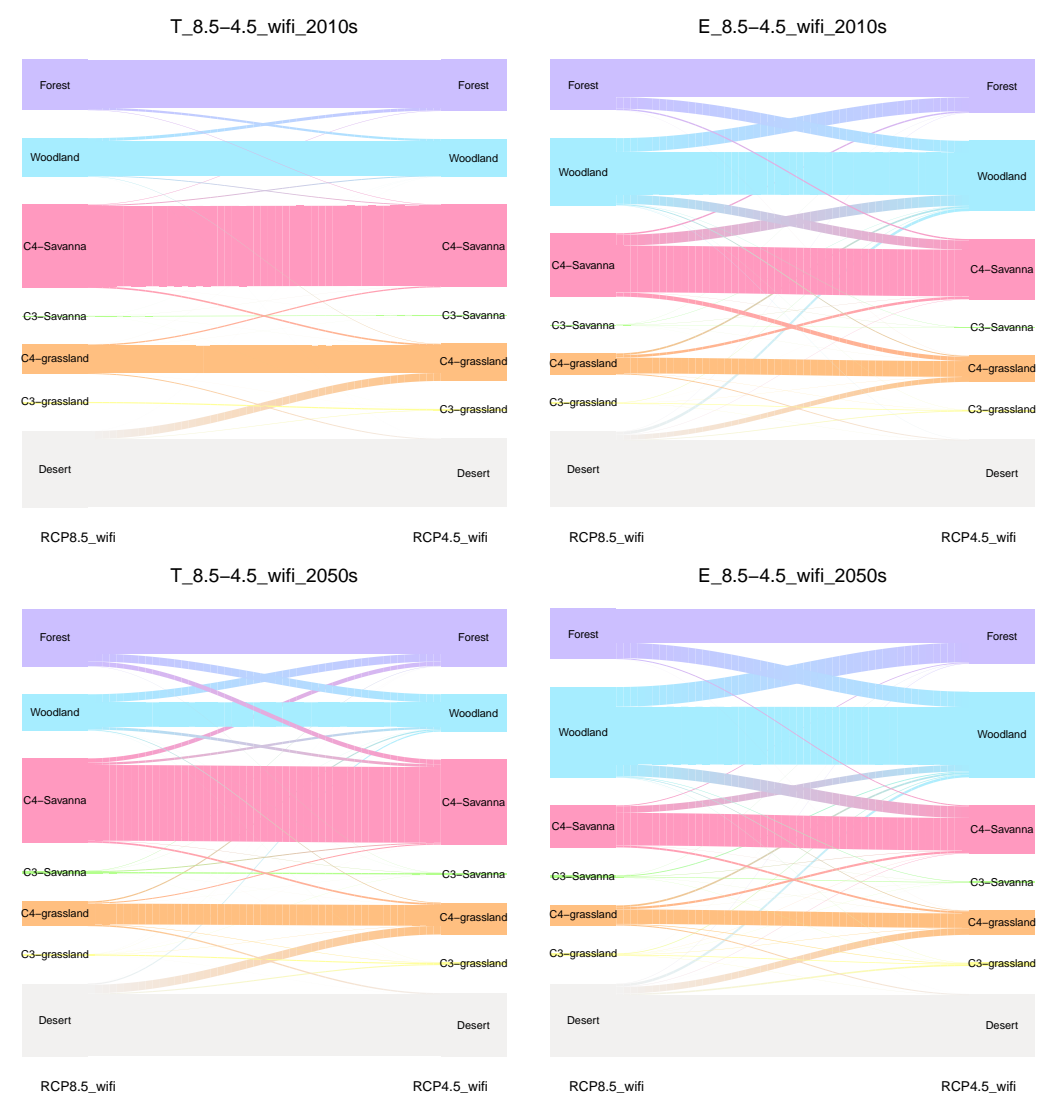

RCP8.5 wifi $\quad$ RCP4.5 wifi

T 8.5-4.5 wifi 2090s

E_8.5-4.5_wifi_2090s
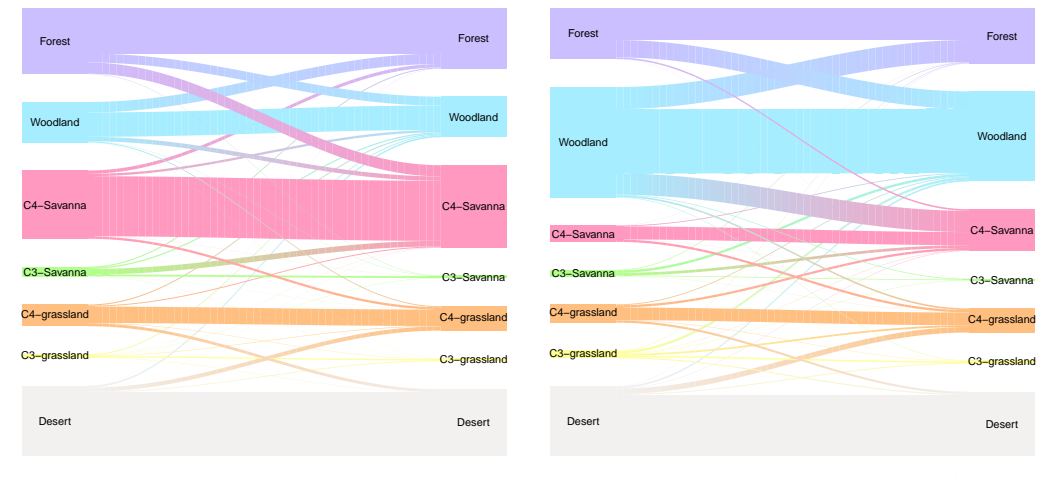

RCP8.5_wifi

RCP4.5_wifi

RCP8.5 wif

RCP4.5_wifi

Figure B7. Sankey diagrams showing the difference in biome type between RCP8.5 and RCP4.5 with fire, for the three decades of interest (2010s, 2050s, 2090s). ”T_ “denotes transient scenarios, ”E_ denotes equilibrium scenarios. 


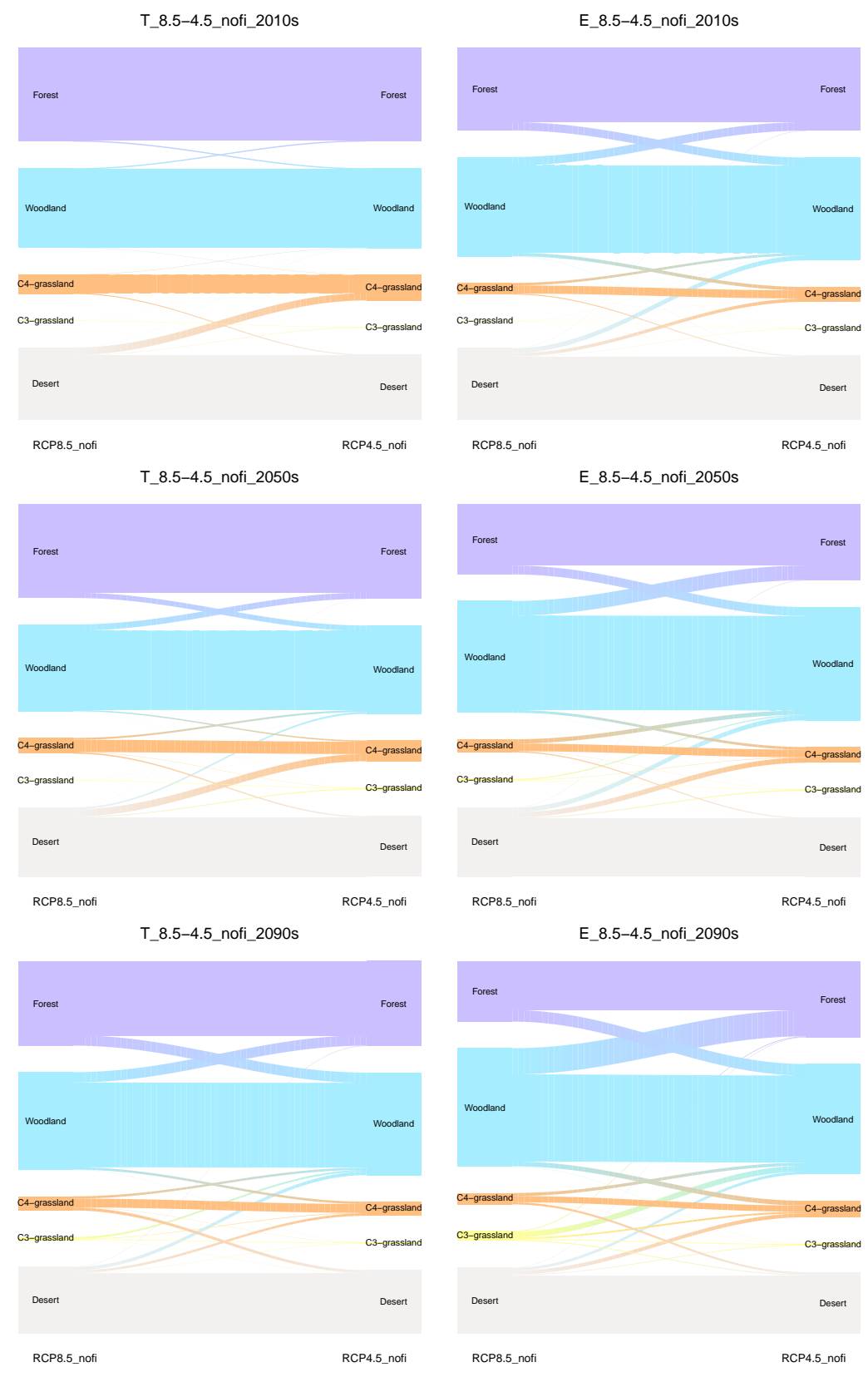

Figure B8. Sankey diagrams showing the difference in biome type between RCP8.5 and RCP4.5 without fire, for the three decades of interest (2010s, 2050s, 2090s). "T_“ denotes transient scenarios, ”E_“ denotes equilibrium scenarios. 


\section{SUPPLEMENT C}

Additional video material supplementing this publication is available and can be downloaded here:

https://data.mendeley.com/datasets/yx8wj84bd2/draft?a=4203fa29-d8bb-4ba2-b96a-8c5ba573facc

The videos show decadal time series of results in form of maps and allow detailed visual comparison of scenarios. 